\title{
Dynamic Analysis of Multi-Storey Building with Openings in Shear Wall
}

\section{Mahdi hosseini $^{1}$, Ahmed Najm Abdullah Al-Askari ${ }^{2}$}

1 Ph.D. scholar in Structural Engineering, Dept. of Civil Engineering, Jawaharlal Nehru Technological University Hyderabad (JNTUH), Hyderabad, Telangana, India

2 Master of Technology in Infrastructure Engineering, Department of Civil Engineering Jawaharlal Nehru Technological University Hyderabad (JNTUH), Telangana, India

\section{ABSTRACT}

Shear walls are added to the building interior to provide extra strength and stiffness to the building when the exterior walls cannot provide sufficient strength and stiffness or when the allowable span-width ratio for the floor or roof diaphragm is exceeded. Shear walls are analyzed to resist two types of forces: shear forces and uplift forces. Shear forces are created throughout the height of the wall between the top and bottom shear wall connections. Uplift forces exist on shear walls because the horizontal forces are applied to the top of the wall. These uplift forces try to lift up one end of the wall and push the other end down. In some cases, the uplift force is large enough to tip the wall over. Shear walls are analyzed to the provide necessary lateral strength to resist horizontal forces. Shear walls are strong enough, to transfer these horizontal forces to the next element in the load path below them. In present work, twenty storey buildings have been modeled using software package ETABS for earthquake zone III in India. This paper aims to study the behavior of reinforced concrete building by conducting dynamic analysis for shear wall with opening conditions. Symmetrical openings are provided in shear walls with proper sizes to ensure least interruption to force flow through walls. Estimation of structural response such as; storey displacements, Torsion ,Bending Moment, storey drift is carried out. Dynamic responses under zone III earthquake as per IS 1893 (part 1) : 2002 have been carried out.

Shear walls are analyzed for two cases

1) With openings

2) Without openings

and the results are compared and tabulated.

Keywords: dynamic analysis, structural response ,shear walls with opening, shear forces , uplift forces

\section{INTRODUCTION}

Shear walls are a type of structural system that provides lateral resistance to a building or structure. They resist in-plane loads that are applied along its height. The applied load is generally transferred to the wall by a diaphragm or collector or drag member. The efficiency of a structural system is measured in terms of their ability to resist lateral load, which increases with the height of the frame. A building can be considered as tall when the effect of lateral loads is reflected in the design. Lateral deflections of framed buildings should be limited to prevent damage to both structural and nonstructural elements. Reinforced concrete 
(RC) buildings often have vertical plate-like RC walls called Shear Walls in addition to slabs, beams and columns. These walls generally start at foundation level and are continuous throughout the building height. Their thickness can be as low as $150 \mathrm{~mm}$, or as high as $400 \mathrm{~mm}$ in high rise buildings. Shear walls are usually provided along both length and width of buildings. Shear walls are like vertically-oriented wide beams that carry earthquake loads downwards to the foundation. "We cannot afford to build concrete buildings meant to resist severe earthquakes without shear walls." Mark Fintel, a noted consulting engineer in USA Shear walls in high seismic regions requires special detailing. However, in past earthquakes, even buildings with sufficient amount of walls that were not specially detailed for seismic performance (but had enough well-distributed reinforcement) were saved from collapse. Shear wall buildings are a popular choice in many earthquake prone countries, like Chile, New Zealand and USA. Shear walls are easy to construct, because reinforcement detailing of walls is relatively straight-forward and therefore easily implemented at site. Shear walls are efficient; both in terms of construction cost properly designed and detailed buildings with Shear walls have shown very good performance in past earthquakes. The overwhelming success of buildings with shear walls in resisting strong earthquakes is summarized in the quote: And effectiveness in minimizing earthquake damage in structural and non- Structural elements (like glass windows and building contents). Shear walls provide large strength and stiffness to buildings in the direction of their orientation, which significantly reduces lateral sway of the building and thereby reduces damage to structure and its contents. Shear walls should be provided along preferably both length and width.Door or window openings can be provided in shear walls, but their size must be small to ensure least interruption to force flow through walls. Moreover, openings should be symmetrically located. Special design checks are required to ensure that the net cross sectional area of a wall at an opening is sufficient to carry the horizontal earthquake force. Shear walls in buildings must be symmetrically located in plan to reduce ill-effects of twist in buildings. They could be placed symmetrically along one or both directions in plan. In modern tall buildings, shear walls are commonly used as a vertical structural element for resisting the lateral loads that may be induced by the effect of wind and earthquakes. Shear walls of varying cross sections i.e. rectangular shapes to more irregular cores such as channel, T, L, barbell shape, box etc. can be used. Provision of walls helps to divide an enclose space, whereas of cores to contain and convey services such as elevator. Wall openings are inevitably required for windows in external walls and for doors or corridors in inner walls or in lift cores. The size and location of openings may vary from architectural and functional point of view.

When a building is subjected to wind or earthquake load, various types of failure must be prevented:

- slipping off the foundation (sliding)

- overturning and uplift (anchorage failure)

- shear distortion (drift or racking deflection)

- collapse (excessive racking deflection)

The first three types of failure are schematically shown in the Figure 1 Clearly, the entire system must be tied together to prevent building collapse or significant deformation. 


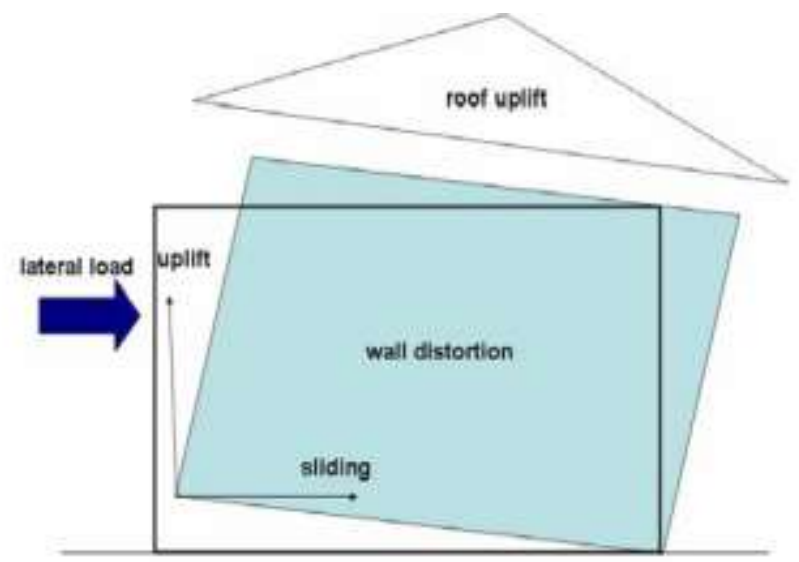

Figure 1: Schematic of the deformations of the structure due to the lateral loads

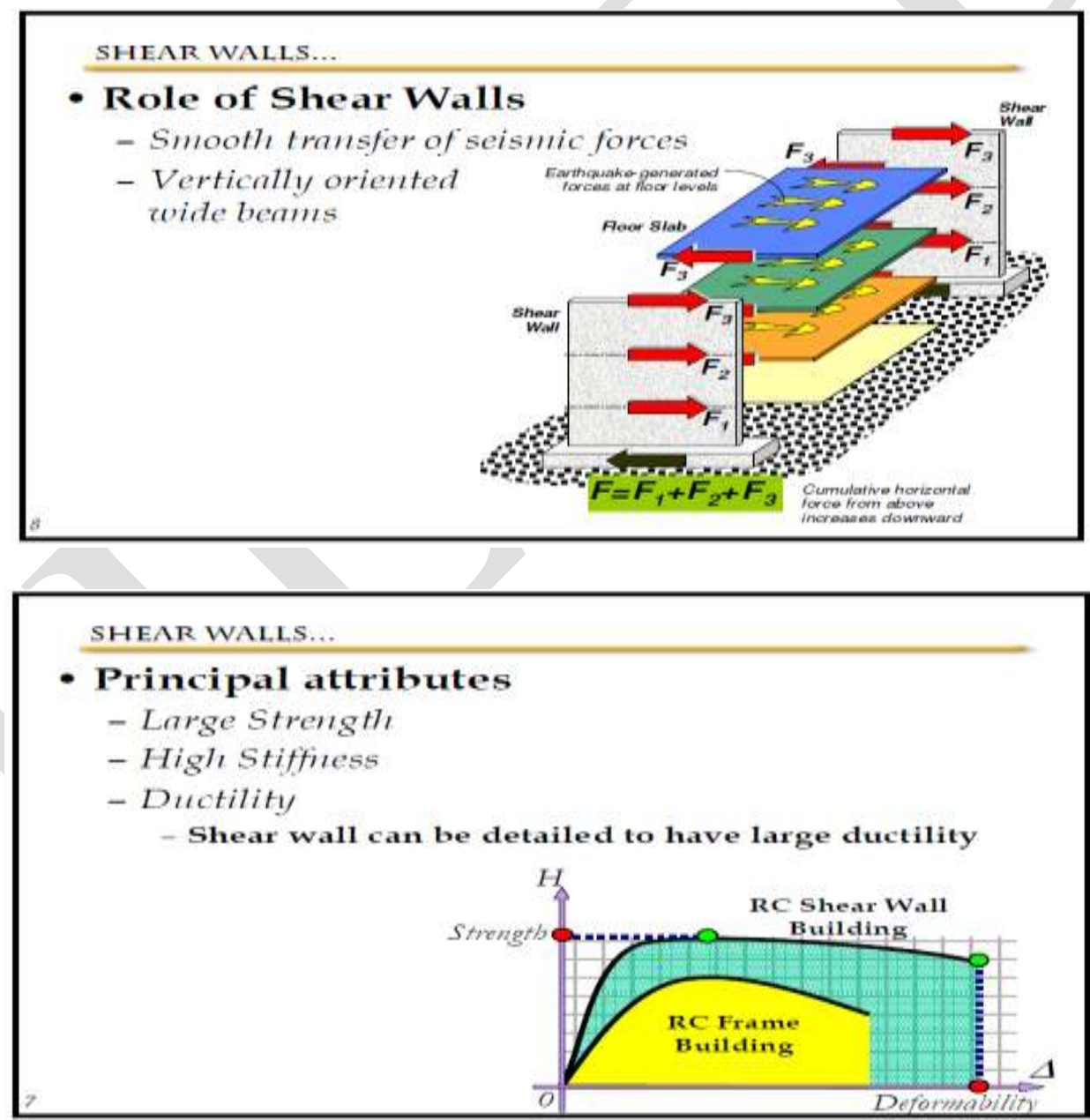


SHEAR WALLS...

- Advantages of Shear Walls...

- Lesser lateral displacement than frames

- Lesser Damage to structural and non-structural elements
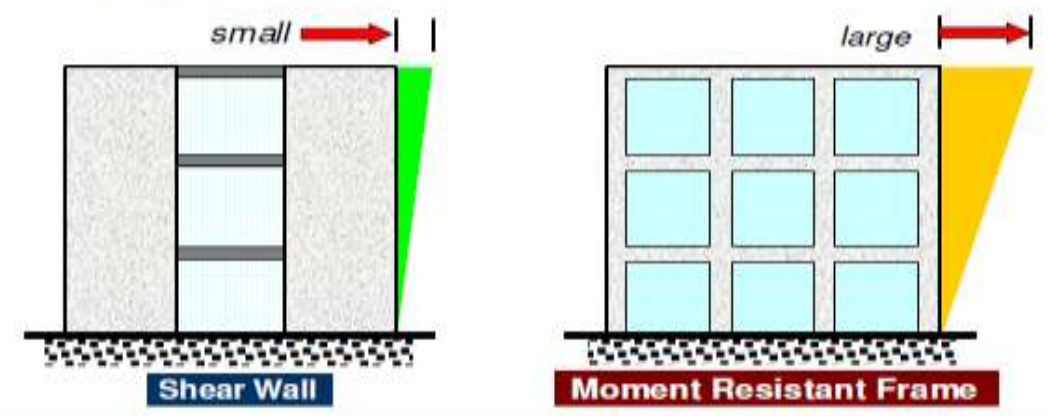

ARCHITECTURAL ASTECTS..

- Openings in walls must be

- As feze as possible

- As small as possible

- As symmetric as possible

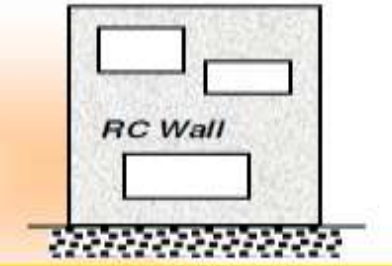

Large and randomly placed openings not allowed

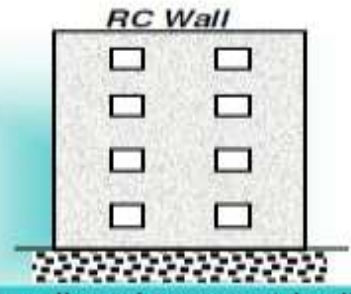

SEISMIC BEHAVIOUR..

- At each section along the height, shear wall carries

- Axial Force P

- Shear Force V

- Bending Moment M

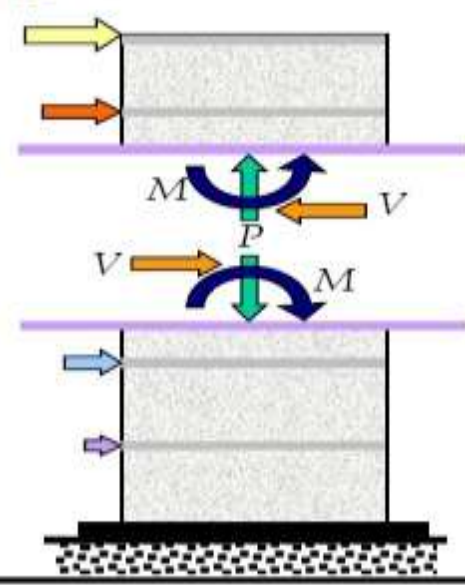




\subsection{Shear Walls with Openings}

Pipers in a wall formed by openings may be regarded as fixed at both ends, which changes the bending deflection tremh/3EI to h3/12EI in esq. the rigidity of a pier is then given in the direction of its length.

$$
R=\frac{E_{t}}{\left(\frac{h}{L}\right)^{3}+2.64\left(\frac{h}{L}\right)}
$$

VALUES OF $(\mathrm{h} / \mathrm{L})$
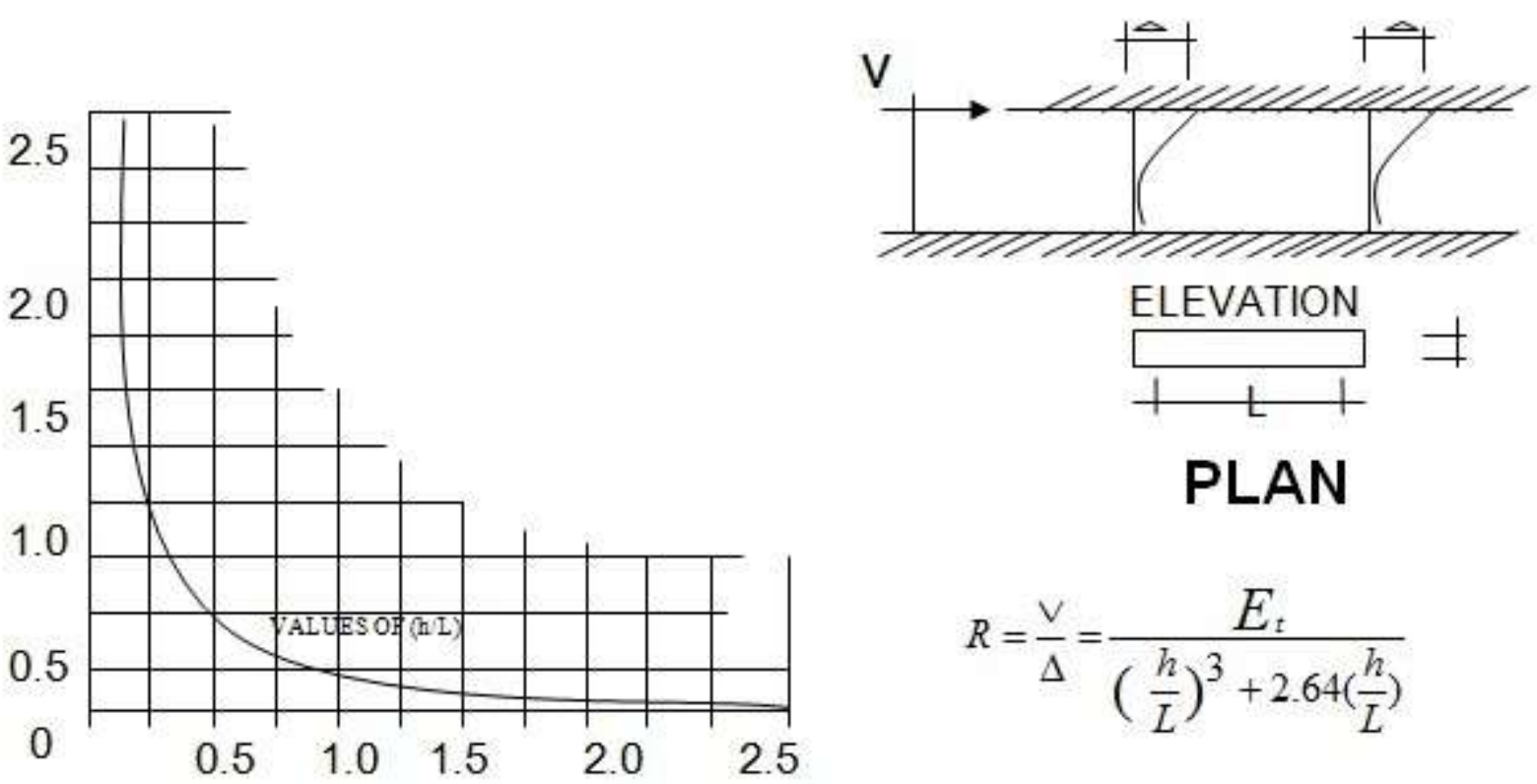

$$
R=\frac{\vee}{\Delta}=\frac{E_{t}}{\left(\frac{h}{L}\right)^{3}+2.64\left(\frac{h}{L}\right)}
$$

Rigidity of wall element fixed at both ends. It gives a curve for rapid evaluations of the rigidity of piers. The rigidity of a pier in the direction of its thickness in negligibly small.

The rigidity of a wall with openings may be calculated neglecting the effect of the axial shortening of piers by the judicious use of the principles of series and parallels in the same way. It is seen that for normal window or door openings, the rigidity of the wall is not affected to any appreciable extent. The rigidity of a shear wall is due more to its form than it its mass.

In size of the openings should be relatively small and these should be spaced at least a distance equal to the size of the openings in each direction. To restrict the stresses in the shear wall, the width of openings should be limited approximately to $15 \%$ of the total length of the connected shear walls and the depth of the connecting beam should be greater than $20 \%$ of the storey height.

\subsection{Rigidity of a Wall Element}

Ryx is defined as the horizontal force necessary to prevent $y$ - distortions of a wall element when $\mathrm{Rx}$ is applied in the x-directions producing a unit deflection Rxy is also defined. When the principal axes of the shape of a shear wall are parallel to the $\mathrm{x}$ and $\mathrm{y}$ axes, Rxy and Ryx vanish. 


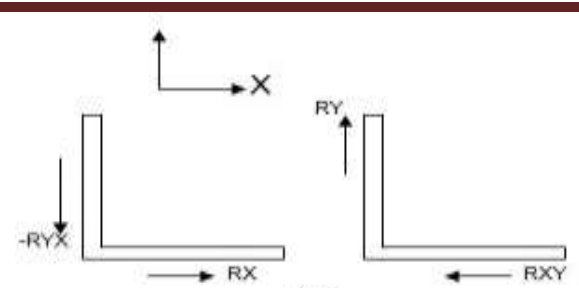

(a)

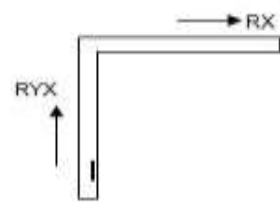

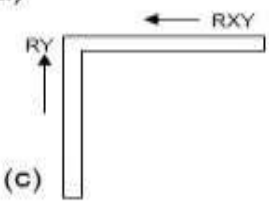

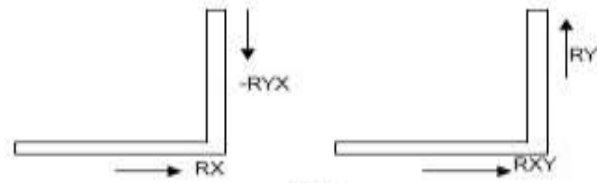

(b)
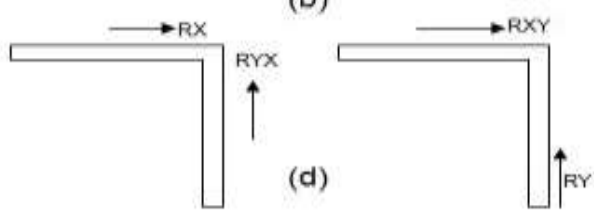

Direction of Rxy and Ryx for various dispositions of the angle wall element. (a) angle in position 1; (b) angle in position 2; (c) angle in position 3; (d) angle in position 4.

\section{METHODOLOGY}

\subsection{Dynamic Analysis}

Dynamic analysis shall be performed to obtain to design seismic force, and its distribution to different levels along the height of the building and to various lateral loads resisting elements for the following buildings:

Regular buildings-those greater than $40 \mathrm{~m}$ in height in zones 4 and 5, those greater than $90 \mathrm{~m}$ in height in zones 2 and 3. The analysis model for dynamic analysis of buildings with unusual configuration should be such that it adequately models the types of irregularities present in the building configuration. Buildings with plan irregularities (as defined in the Table 4 of IS 1893-2002) can not be modeled for dynamic analysis.

Dynamic analysis may be performed either by the time history method or by the response spectrum method. However, in either method, the design base shear $\left(\bar{V}_{B}\right)$ shall be compared with a base shear $\left(V_{B}\right)$ calculated using a fundamental period t. where $\left(\bar{V}_{B}\right)$ is less than $\left(V_{B}\right)$, all response quantities (for example member forces, displacements, storey forces, storey shears and base reactions) shall be multiplied by $\left(V_{B}\right) /\left(\bar{V}_{B}\right)$. The values of damping for building may be taken as 2 and 5 percent of the critical, for the purposes of dynamic analysis of steel and reinforce concrete buildings, respectively.

a) Time history method- the usage of this method shall be on an appropriate ground motion and shall be performed using accepted principles of dynamics.

b) Response spectrum method- this method shall be performed using the design spectrum specified in code or by a site-specific design spectrum for a structure prepared at a project site.

\section{NUMERICAL MODELLING AND ANALYSIS}

\subsection{Geometrical Properties}

1. No. of stories of the Building model $=20$

2. Column Sizes: -

a) Outer periphery columns $=600 \mathrm{~mm} \times 600 \mathrm{~mm}$

3. Beam Size $=400 \mathrm{~mm} \times 600 \mathrm{~mm}$

4. Slab thickness $=150 \mathrm{~mm}$ 


\subsection{Loads Calculations}

1. Live load
a) Corridor $=3 \mathrm{KN} / \mathrm{m} 2$
b) Floor $=2 \mathrm{KN} / \mathrm{m} 2$

2. Dead Load (Floor Finishing) $=1.5 \mathrm{KN} / \mathrm{m} 2$

3. Wall load
a) $9^{\prime \prime}=12.4 \mathrm{KN} / \mathrm{m}$
b) $41 / 2 "=7 \mathrm{KN} / \mathrm{m}$

4. Wind load

a) Wind Exposure parameters

Wind direction angle $=0$ Degree

Windward coff. $\mathrm{Cp}=0.8$

Leeward coff $\mathrm{Cp} \quad=0.5$

b) Wind coefficients

Wind speed

$$
=50 \mathrm{KN} / \mathrm{m}
$$

Terrain category $\quad=2$

Structure class $=\mathrm{B}$

Risk coefficient $(\mathrm{k} 1) \quad=1$

Topography $(\mathrm{k} 3)=1$

5. Seismic loading

Seismic zone factor $(\mathrm{Z}) \quad=0.16$

Soil Type $\quad=$ Medium (II)

Response Reduction factor $=5$

\subsection{Load Combinations}

The following Load Combinations have been considered for the design

$$
\begin{aligned}
& \text { (DL+ LL) } \\
& \text { (DL } \pm \text { EQXTP) } \\
& \text { (DL } \pm \text { EQYTP) } \\
& \text { (DL } \pm \text { EQXTN) } \\
& \text { (DL } \pm \text { EQYTN) } \\
& \text { (DL + LL } \pm \text { EQXTP) } \\
& \text { (DL + LL } \pm \text { EQYTP) } \\
& \text { (DL }+\mathrm{LL} \pm \mathrm{EQXTN}) \\
& (\mathrm{DL}+\mathrm{LL} \pm \mathrm{EQYTN}) \\
& \text { (DL } \pm \text { WLX) } \\
& \text { (DL } \pm \text { WLY) } \\
& (\mathrm{DL}+\mathrm{LL} \pm \mathrm{WLX}) \\
& (\mathrm{DL}+\mathrm{LL} \pm \mathrm{WLY}) \\
& 1.5(\mathrm{DL}+\mathrm{LL}) \\
& 1.5(\mathrm{DL} \pm \mathrm{EQXTP}) \\
& 1.5(\mathrm{DL} \pm \mathrm{EQYTP}) \\
& 1.5(\mathrm{DL} \pm \mathrm{EQXTN}) \\
& 1.5(\mathrm{DL} \pm \mathrm{EQYTN}) \\
& 1.2(\mathrm{DL}+\mathrm{LL} \pm \mathrm{EQXTP}) \\
& \text { 1.2(DL + LL } \pm \text { EQYTP) } \\
& 1.2(\mathrm{DL}+\mathrm{LL} \pm \text { EQXTN }) \\
& \text { 1.2(DL + LI } \pm \text { EQYTN }) \\
& \text { 1.5(DL } \pm \text { WLX }) \\
& 1.5(\mathrm{DL} \pm \mathrm{WLY}) \\
& \text { 1.2(DL }+\mathrm{LL} \pm \mathrm{WLX}) \\
& 1.2(\mathrm{DL}+\mathrm{LL} \pm \mathrm{WLY})
\end{aligned}
$$




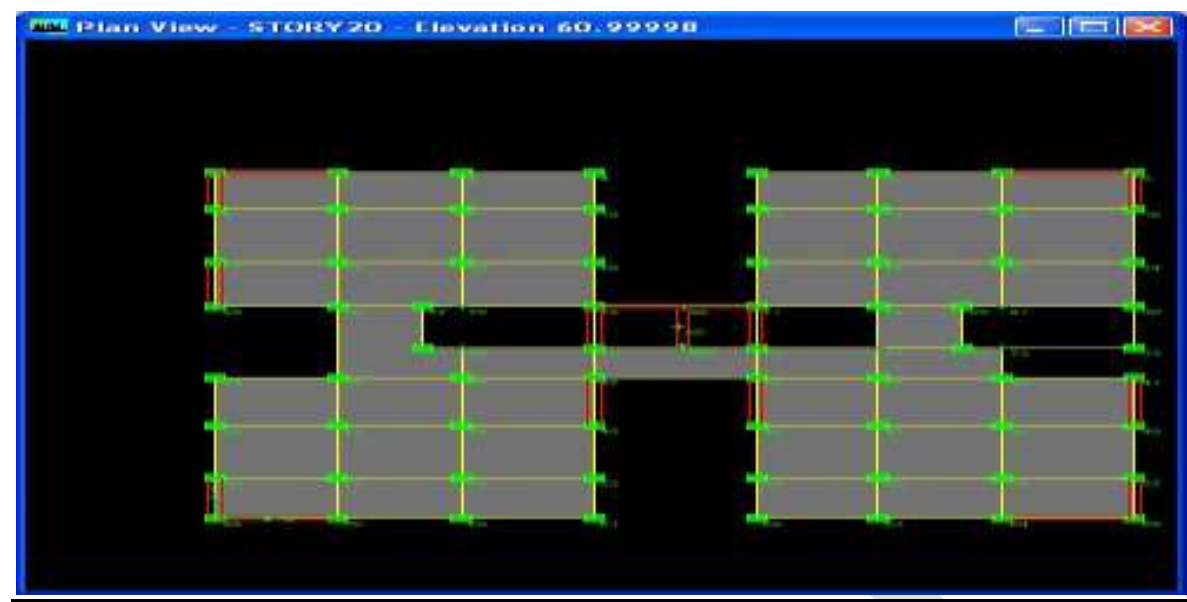

Figure 2: PLAN

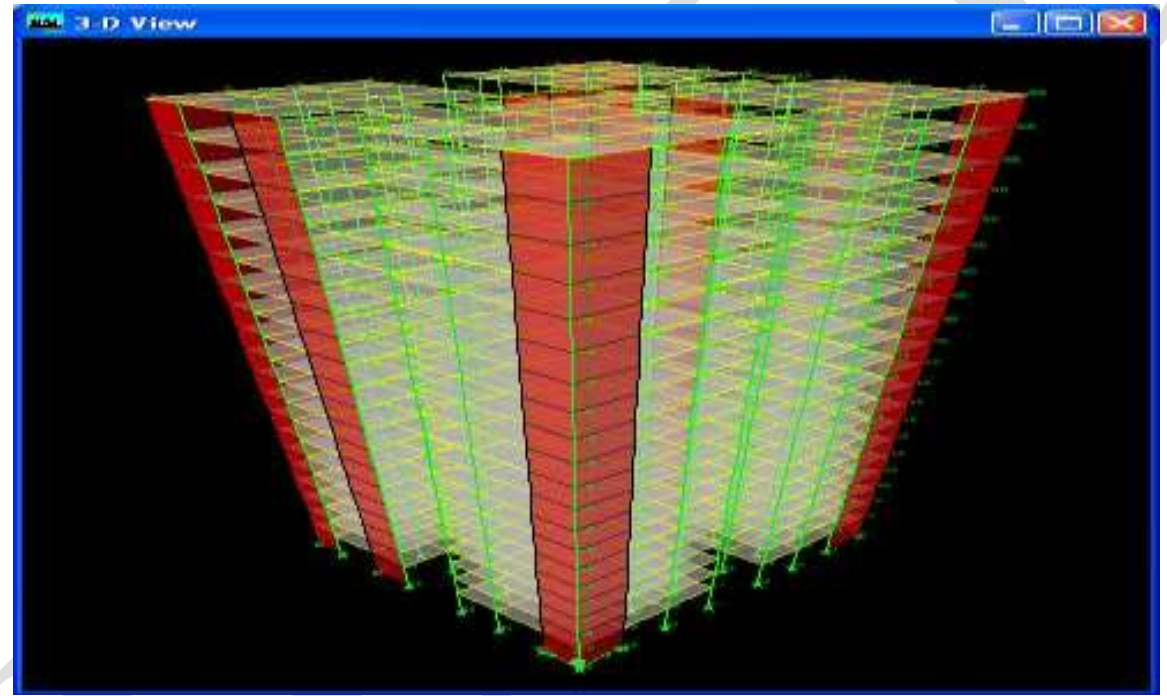

Figure 3: 3-D MODEL OF A BUILDING

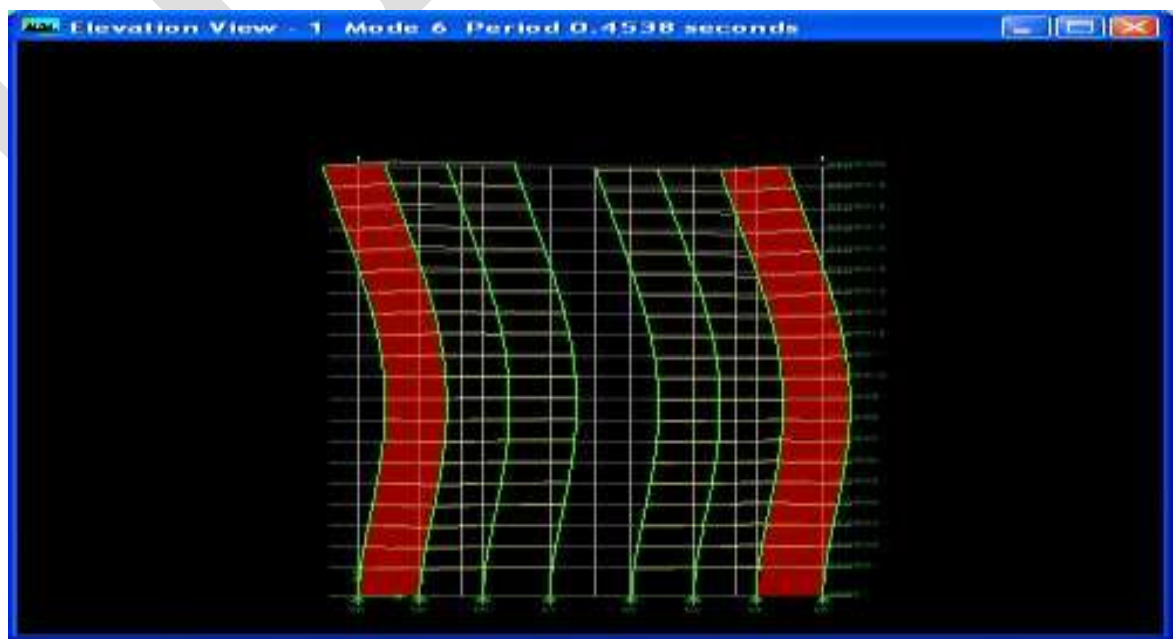

Figure 4: MODE SHAPE - SHEAR WALL WITHOUT OPENING 


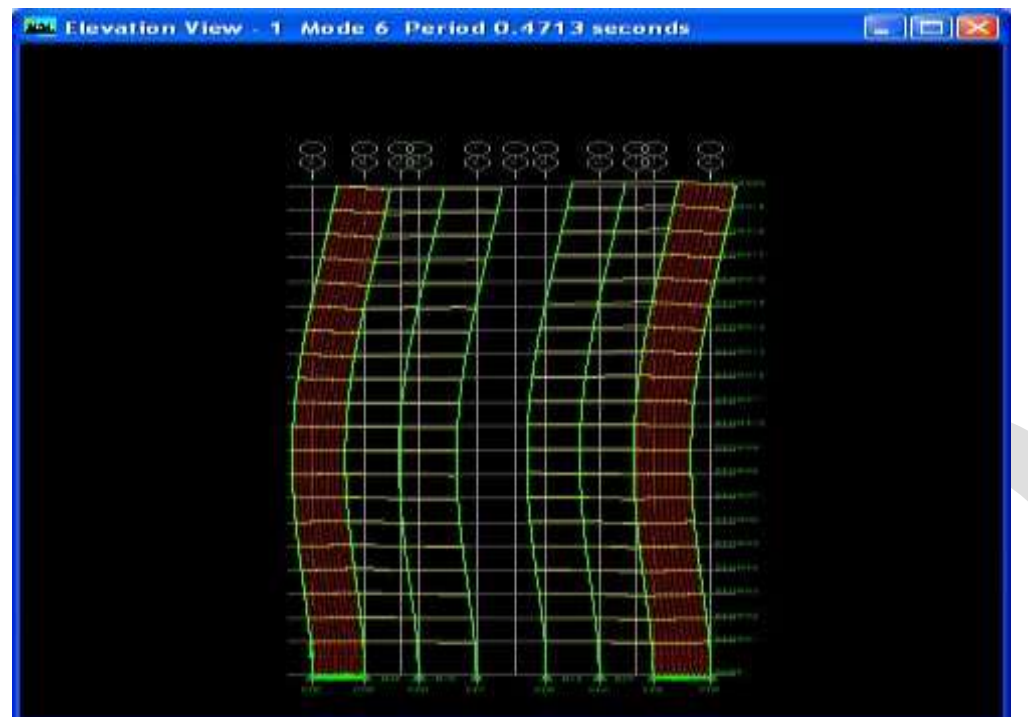

Figure 5: MODE SHAPE - 6 SHEAR WALL WITH OPENING

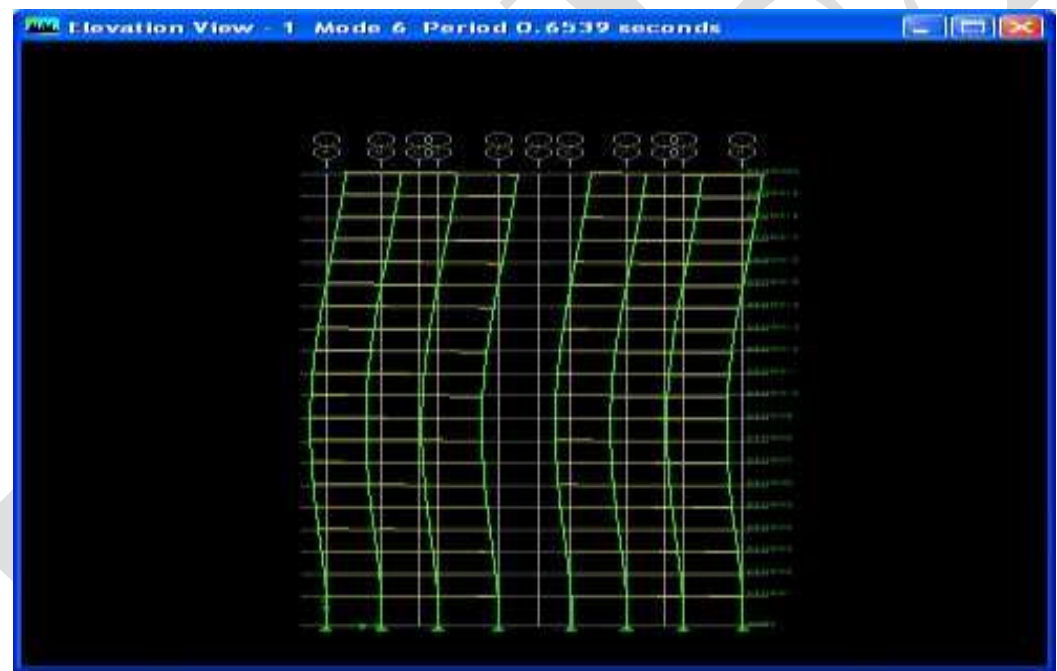

Figure 6: MODE SHAPE - 6 WITHOUT SHEAR WALL

\section{RESULTS AND DISCUSSIONS}

$\%$ Torsion of shear wall without opening is compared with different $\%$ openings and tabulated for column $\mathrm{C} 85$, which is nearer to shear wall

\begin{tabular}{|c|c|c|}
\hline$\%$ opening & $\begin{array}{c}\text { For single opening the \% } \\
\text { increase in Torsion }\end{array}$ & $\begin{array}{c}\text { For two openings the \% } \\
\text { increase in Torsion }\end{array}$ \\
\hline $20 \%$ & 37.6 & 14.4 \\
\hline $30 \%$ & 39.5 & 17.5 \\
\hline $40 \%$ & 39.4 & 20.43 \\
\hline
\end{tabular}


TABLE 1: Torsion for column C85 which is nearer to shear wall

\begin{tabular}{|c|c|c|c|c|}
\hline STORY & $\begin{array}{c}\text { without } \\
\text { SW }\end{array}$ & $20 \%$ & $30 \%$ & $40 \%$ \\
\hline STORY1-21 & -3.169 & -4.424 & -4.198 & -4.577 \\
\hline STORY1-20 & -3.202 & -4.384 & -4.235 & -4.847 \\
\hline STORY1-19 & -3.122 & -4.327 & -4.271 & -5.117 \\
\hline STORY1-18 & -2.987 & -4.232 & -4.271 & -5.352 \\
\hline STORY1-17 & -2.812 & -4.114 & -4.239 & -5.546 \\
\hline STORY1-16 & -2.607 & -3.978 & -4.184 & -5.697 \\
\hline STORY1-15 & -2.382 & -3.828 & -4.107 & -5.807 \\
\hline STORY1-14 & -2.143 & -3.662 & -4.01 & -5.876 \\
\hline STORY1-13 & -1.896 & -3.482 & -3.891 & -5.905 \\
\hline STORY1-12 & -1.645 & -3.284 & -3.751 & -5.894 \\
\hline STORY1-11 & -1.394 & -3.069 & -3.588 & -5.841 \\
\hline STORY1-10 & -1.146 & -2.835 & -3.403 & -5.747 \\
\hline STORY1-9 & -0.906 & -2.582 & -3.194 & -5.613 \\
\hline STORY1-8 & -0.681 & -2.311 & -2.965 & -5.441 \\
\hline STORY1-7 & -0.475 & -2.026 & -2.718 & -5.234 \\
\hline STORY1-6 & -0.299 & -1.731 & -2.459 & -4.998 \\
\hline STORY1-5 & -0.164 & -1.435 & -2.2 & -4.738 \\
\hline STORY1-4 & -0.084 & -1.153 & -1.952 & -4.456 \\
\hline STORY1-3 & -0.08 & -0.911 & -1.744 & -4.141 \\
\hline STORY1-2 & -0.162 & -0.718 & -1.57 & -3.709 \\
\hline STORY1-1 & -0.397 & -0.759 & -1.534 & -3.109 \\
\hline STORY1 & -0.762 & -0.357 & -0.658 & -1.135 \\
\hline
\end{tabular}

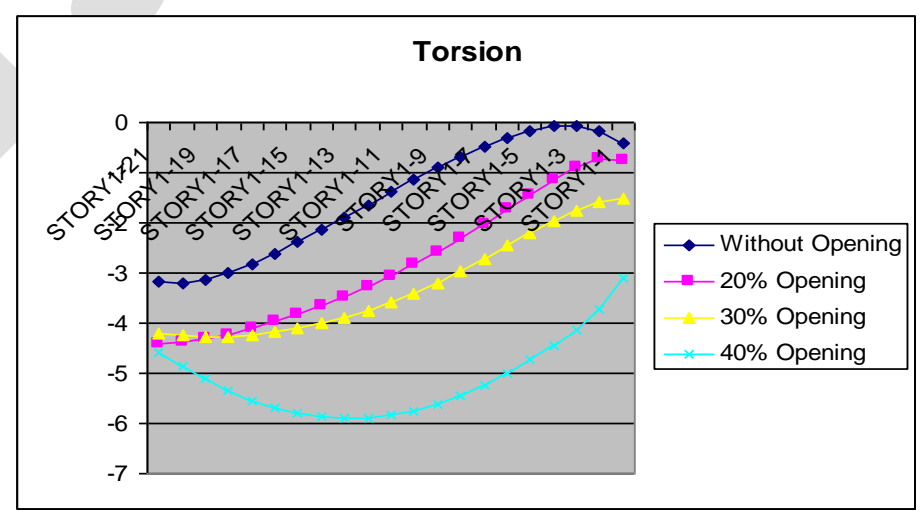

Graph 1: Torsion for C85 column which is nearer to the shear wall 
$\%$ Shear Force of shear wall without opening is compared with different \% openings and tabulated for column $\mathrm{C} 85$, which is nearer to shear wall

\begin{tabular}{|c|c|c|}
\hline \% opening & $\begin{array}{c}\text { For single opening the \% } \\
\text { increase in Shear Force }\end{array}$ & $\begin{array}{c}\text { For two openings the \% } \\
\text { increase in Shear Force }\end{array}$ \\
\hline $20 \%$ & 16.4 & 17.1 \\
\hline $30 \%$ & 17.24 & 18.6 \\
\hline $40 \%$ & 18.5 & 19.3 \\
\hline
\end{tabular}

TABLE 2: Shear Force for column C85 which is nearer to shear wall

\begin{tabular}{|c|c|c|c|c|}
\hline STORY & without & $20 \%$ & $30 \%$ & $40 \%$ \\
\hline STORY1-21 & -4.95 & -12.61 & -1.9 & 12.64 \\
\hline STORY1-20 & -49.92 & -36.64 & -33.49 & -21.62 \\
\hline STORY1-19 & -50.7 & -43.99 & -41.99 & -35.14 \\
\hline STORY1-18 & -62.93 & -57.01 & -55.85 & -51.19 \\
\hline STORY1-17 & -73 & -69.28 & -68.89 & -65.66 \\
\hline STORY1-16 & -83.18 & -81.14 & -81.38 & -79.14 \\
\hline STORY1-15 & -92.74 & -92.27 & -93.03 & -91.49 \\
\hline STORY1-14 & -101.59 & -102.53 & -103.74 & -102.71 \\
\hline STORY1-13 & -109.58 & -111.83 & -113.44 & -112.8 \\
\hline STORY1-12 & -116.6 & -120.1 & -122.08 & -121.79 \\
\hline STORY1-11 & -122.57 & -127.28 & -129.63 & -129.71 \\
\hline STORY1-10 & -127.4 & -133.33 & -136.08 & -136.58 \\
\hline STORY1-9 & -131 & -138.18 & -141.37 & -142.42 \\
\hline STORY1-8 & -133.25 & -141.76 & -145.45 & -147.26 \\
\hline STORY1-7 & -134.01 & -143.94 & -148.23 & -151.08 \\
\hline STORY1-6 & -133.08 & -144.51 & -149.53 & -153.82 \\
\hline STORY1-5 & -130.23 & -143.32 & -149.24 & -155.57 \\
\hline STORY1-4 & -124.8 & -139.46 & -146.37 & -155.42 \\
\hline STORY1-3 & -117.55 & -134.6 & -143.25 & -156.84 \\
\hline STORY1-2 & -101.22 & -118 & -126.3 & -143.41 \\
\hline STORY1-1 & -90.64 & -108.34 & -116.88 & -134.27 \\
\hline STORY1 & -85.55 & -106.43 & -111.78 & -133.24 \\
\hline
\end{tabular}




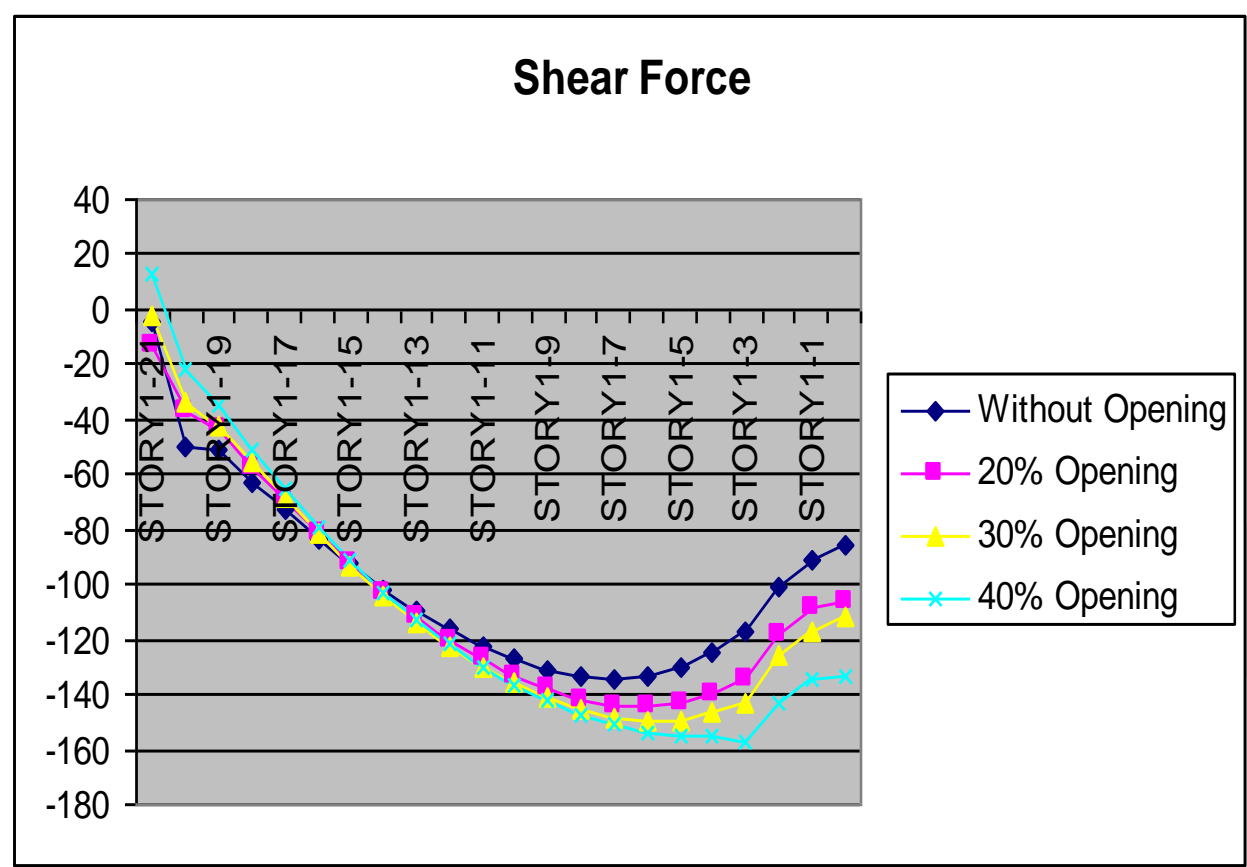

Graph 2: Shear force for C85column which is nearer to the shear wall

$\%$ Bending Moment is tabulated when compared with shear wall without opening for column C85, near to Shear wall

\begin{tabular}{|c|c|c|}
\hline \% opening & $\begin{array}{c}\text { For single opening the \% } \\
\text { increase in Bending Moment }\end{array}$ & $\begin{array}{c}\text { For two openings the \% } \\
\text { increase in Bending Moment }\end{array}$ \\
\hline $20 \%$ & 1.9 & 2.46 \\
\hline $30 \%$ & 2.1 & 2.5 \\
\hline $40 \%$ & 2.17 & 1.5 \\
\hline
\end{tabular}


TABLE 3: Moment for column C85 which is nearer to shear wall

\begin{tabular}{|c|c|c|c|c|}
\hline STORY & without SW & $20 \%$ & $30 \%$ & $40 \%$ \\
\hline STORY1-21 & -7.934 & 15.231 & 31.455 & 48.322 \\
\hline STORY1-20 & -40.58 & -14.32 & -4.165 & 17.864 \\
\hline STORY1-19 & -44.42 & -27.508 & -21.154 & -4.447 \\
\hline STORY1-18 & -58.851 & -45.535 & -41.633 & -29.834 \\
\hline STORY1-17 & -74.218 & -64.91 & -62.857 & -54.768 \\
\hline STORY1-16 & -91.123 & -85.113 & -84.478 & -79.027 \\
\hline STORY1-15 & -108.543 & -105.438 & -105.903 & -102.25 \\
\hline STORY1-14 & -126 & -125.421 & -126.74 & -124.268 \\
\hline STORY1-13 & -143.094 & -144.738 & -146.724 & -144.984 \\
\hline STORY1-12 & -159.545 & -163.173 & -165.687 & -164.361 \\
\hline STORY1-11 & -175.154 & -180.584 & -183.529 & -182.401 \\
\hline STORY1-10 & -189.779 & -196.888 & -200.201 & -199.135 \\
\hline STORY1-9 & -203.328 & -212.04 & -215.687 & -214.614 \\
\hline STORY1-8 & -215.736 & -226.016 & -229.984 & -228.894 \\
\hline STORY1-7 & -226.965 & -238.815 & -243.112 & -242.06 \\
\hline STORY1-6 & -236.949 & -250.356 & -254.987 & -254.093 \\
\hline STORY1-5 & -245.795 & -260.859 & -265.9 & -265.489 \\
\hline STORY1-4 & -252.79 & -269.035 & -274.235 & -274.584 \\
\hline STORY1-3 & -260.993 & -279.991 & -286.525 & -290.446 \\
\hline STORY1-2 & -255.003 & -270.546 & -272.478 & -276.794 \\
\hline STORY1-1 & -254.655 & -269.421 & -271.498 & -270.105 \\
\hline
\end{tabular}

\section{Moment}

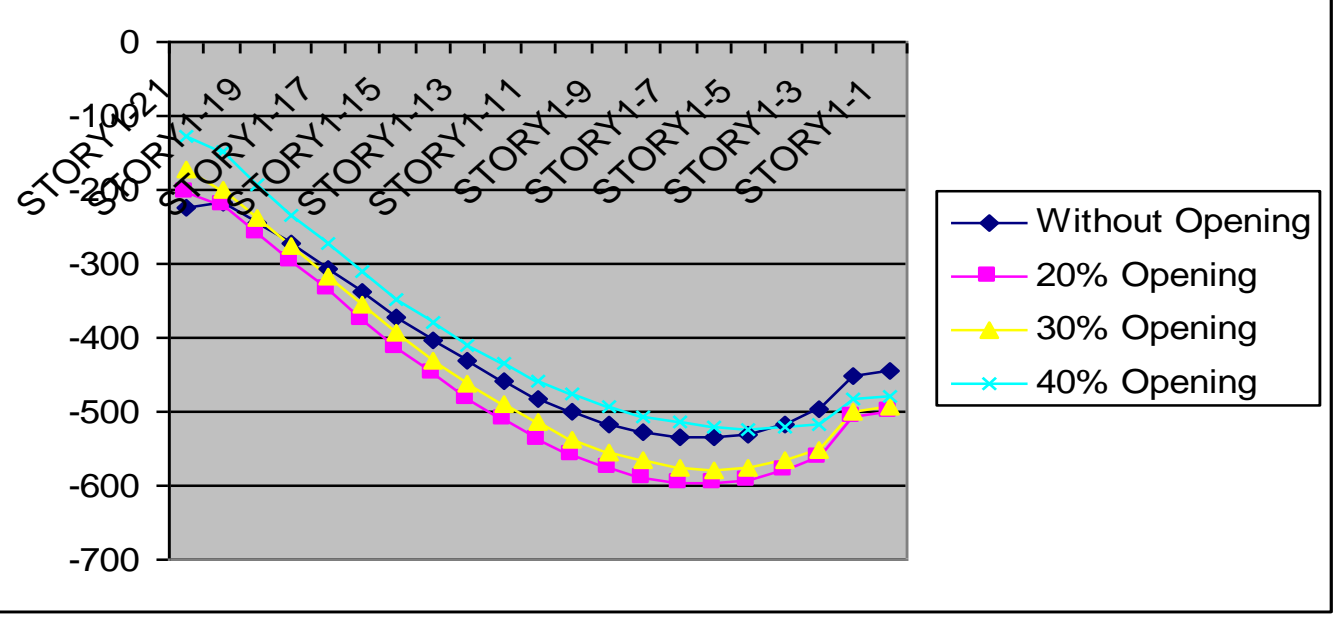

Graph 3: Bending moment for C85 column which is nearer to the shear wall 
DOI : https://dx.doi.org/10.26808/rs.ed.i8v5.03 International Journal of Emerging Trends in Engineering and Development Issue 8, Vol.5 (Aug-Sep 2018) Available online on http://www.rspublication.com/ijeted/ijeted_index.htm ISSN 2249-6149

TABLE 4: Displacement

\begin{tabular}{|l|r|r|r|r|r|r|r|}
\hline & \multicolumn{1}{l}{ without } & & & & & \\
STORY & SW & $20 \%$ & $30 \%$ & $40 \%$ & $20 \% \_2$ & \multicolumn{1}{l|}{$30 \% \_2$} & $40 \% \_2$ \\
\hline STORY1-21 & -0.1557 & -0.1619 & -0.1632 & -0.1631 & -0.1612 & -0.1633 & -0.1651 \\
\hline STORY1-20 & -0.1501 & -0.1566 & -0.1582 & -0.1585 & -0.156 & -0.1583 & -0.1603 \\
\hline STORY1-19 & -0.1442 & -0.1509 & -0.1526 & -0.1534 & -0.1503 & -0.1527 & -0.1549 \\
\hline STORY1-18 & -0.138 & -0.1446 & -0.1465 & -0.1477 & -0.1442 & -0.1467 & -0.149 \\
\hline STORY1-17 & -0.1313 & -0.138 & -0.1399 & -0.1415 & -0.1376 & -0.1401 & -0.1426 \\
\hline STORY1-16 & -0.1242 & -0.1308 & -0.1328 & -0.1347 & -0.1305 & -0.1331 & -0.1356 \\
\hline STORY1-15 & -0.1168 & -0.1233 & -0.1253 & -0.1275 & -0.123 & -0.1256 & -0.1281 \\
\hline STORY1-14 & -0.1089 & -0.1153 & -0.1174 & -0.1197 & -0.1151 & -0.1177 & -0.1202 \\
\hline STORY1-13 & -0.1008 & -0.107 & -0.1091 & -0.1117 & -0.1068 & -0.1094 & -0.112 \\
\hline STORY1-12 & -0.0924 & -0.0984 & -0.1005 & -0.1032 & -0.0983 & -0.1009 & -0.1034 \\
\hline STORY1-11 & -0.0837 & -0.0895 & -0.0917 & -0.0946 & -0.0895 & -0.092 & -0.0946 \\
\hline STORY1-10 & -0.075 & -0.0805 & -0.0827 & -0.0857 & -0.0806 & -0.083 & -0.0856 \\
\hline STORY1-9 & -0.0661 & -0.0714 & -0.0736 & -0.0768 & -0.0715 & -0.0739 & -0.0764 \\
\hline STORY1-8 & -0.0573 & -0.0623 & -0.0645 & -0.0678 & -0.0625 & -0.0648 & -0.0673 \\
\hline STORY1-7 & -0.0487 & -0.0533 & -0.0555 & -0.0588 & -0.0535 & -0.0557 & -0.0582 \\
\hline STORY1-6 & -0.0402 & -0.0445 & -0.0466 & -0.0499 & -0.0448 & -0.0469 & -0.0493 \\
\hline STORY1-5 & -0.0321 & -0.036 & -0.038 & -0.0413 & -0.0363 & -0.0382 & -0.0405 \\
\hline STORY1-4 & -0.0244 & -0.028 & -0.0298 & -0.0329 & -0.0283 & -0.03 & -0.0322 \\
\hline STORY1-3 & -0.0175 & -0.0205 & -0.0222 & -0.025 & -0.0208 & -0.0223 & -0.0243 \\
\hline STORY1-2 & -0.0113 & -0.0138 & -0.0152 & -0.0176 & -0.0141 & -0.0154 & -0.017 \\
\hline STORY1-1 & -0.0061 & -0.008 & -0.0091 & -0.0108 & -0.0083 & -0.0092 & -0.0105 \\
\hline STORY1 & -0.0024 & -0.0024 & -0.0041 & -0.005 & -0.0037 & -0.0042 & -0.0049 \\
\hline
\end{tabular}

\section{Displacement Graph}

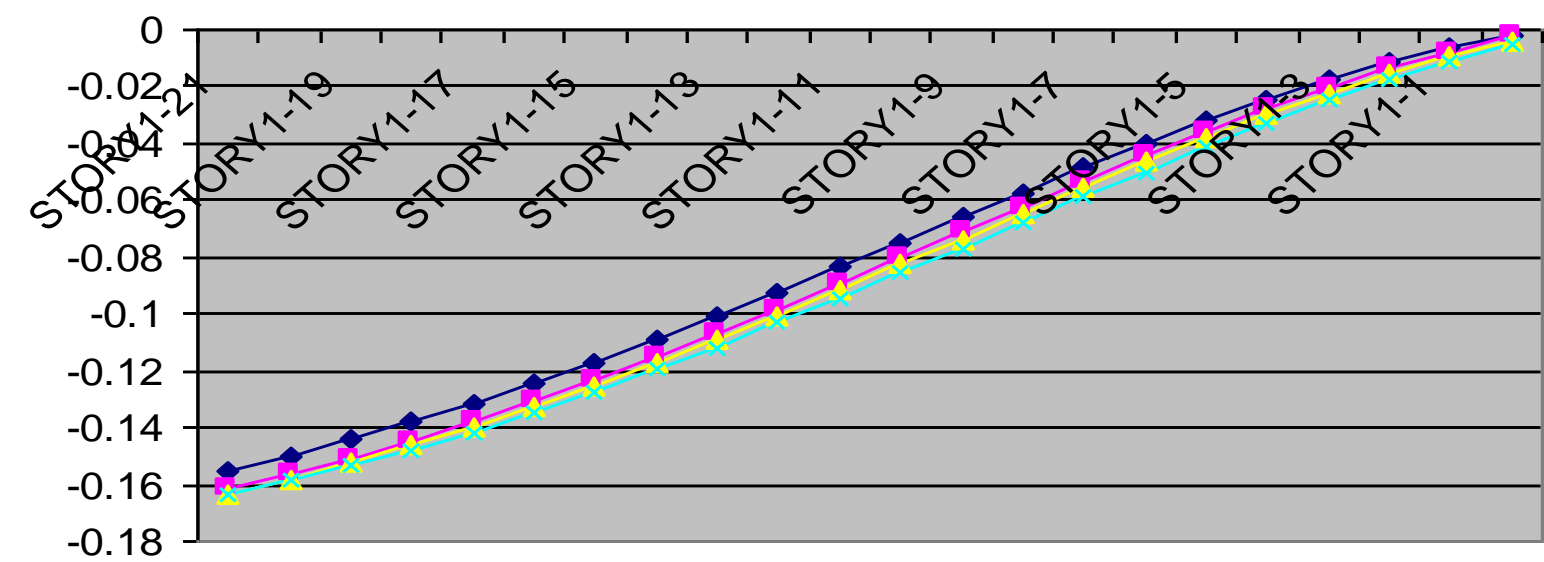


DOI : https://dx.doi.org/10.26808/rs.ed.i8v5.03

International Journal of Emerging Trends in Engineering and Development

Issue 8, Vol.5 (Aug-Sep 2018)

Available online on http://www.rspublication.com/ijeted/ijeted_index.htm

ISSN 2249-6149

TABLE 5: Drift

\begin{tabular}{|c|c|c|c|c|c|c|c|}
\hline STORY & $\begin{array}{l}\text { without } \\
\text { SW }\end{array}$ & $20 \%$ & $30 \%$ & $40 \%$ & $20 \% \_2$ & $30 \% \_2$ & $40 \% \_2$ \\
\hline STORY1-21 & 0.001929 & 0.001929 & 0.001938 & 0.001801 & 0.00196 & 0.001918 & 0.001857 \\
\hline STORY1-20 & 0.002037 & 0.002037 & 0.002101 & 0.001995 & 0.002108 & 0.002081 & 0.002041 \\
\hline STORY1-19 & 0.002153 & 0.002153 & 0.002264 & 0.002186 & 0.002259 & 0.002244 & 0.00222 \\
\hline STORY1-18 & 0.002281 & 0.002281 & 0.002424 & 0.002366 & 0.00241 & 0.002405 & 0.002392 \\
\hline STORY1-17 & 0.002411 & 0.002411 & 0.002575 & 0.002533 & 0.002553 & 0.002557 & 0.002552 \\
\hline STORY1-16 & 0.002538 & 0.002538 & 0.002712 & 0.002681 & 0.002685 & 0.002695 & 0.002697 \\
\hline STORY1-15 & 0.002655 & 0.002655 & 0.002833 & 0.002811 & 0.002801 & 0.002816 & 0.002824 \\
\hline STORY1-14 & 0.002758 & 0.002758 & 0.002934 & 0.002919 & 0.002898 & 0.002919 & 0.00293 \\
\hline STORY1-13 & 0.002844 & 0.002844 & 0.003014 & 0.003006 & 0.002973 & 0.002999 & 0.003014 \\
\hline STORY1-12 & 0.002909 & 0.002909 & 0.00307 & 0.003068 & 0.003026 & 0.003055 & 0.003074 \\
\hline STORY1-11 & 0.00295 & 0.00295 & 0.003101 & 0.003107 & 0.003052 & 0.003087 & 0.003111 \\
\hline STORY1-10 & 0.002966 & 0.002966 & 0.003109 & 0.003121 & 0.003057 & 0.003095 & 0.003124 \\
\hline STORY1-9 & 0.002953 & 0.002953 & 0.0031 & 0.003114 & 0.003048 & 0.003088 & 0.003116 \\
\hline STORY1-8 & 0.002908 & 0.002908 & 0.00307 & 0.003098 & 0.003014 & 0.00306 & 0.003094 \\
\hline STORY1-7 & 0.002829 & 0.002829 & 0.003008 & 0.003054 & 0.002946 & 0.002999 & 0.003043 \\
\hline STORY1-6 & 0.00271 & 0.00271 & 0.002911 & 0.002981 & 0.002842 & 0.002904 & 0.002959 \\
\hline STORY1-5 & 0.002548 & 0.002548 & 0.002774 & 0.002877 & 0.002697 & 0.002769 & 0.00284 \\
\hline STORY1-4 & 0.002334 & 0.002334 & 0.002592 & 0.002739 & 0.002505 & 0.002589 & 0.00268 \\
\hline STORY1-3 & 0.00206 & 0.00206 & 0.002359 & 0.00256 & 0.002258 & 0.002356 & 0.002475 \\
\hline STORY1-2 & 0.001713 & 0.001713 & 0.002063 & 0.002326 & 0.001945 & 0.002059 & 0.002213 \\
\hline STORY1-1 & 0.001271 & 0.001271 & 0.001706 & 0.002024 & 0.001568 & 0.001708 & 0.001909 \\
\hline STORY1 & 0.000688 & 0.000688 & 0.001183 & 0.00144 & 0.00109 & 0.001255 & 0.001443 \\
\hline
\end{tabular}

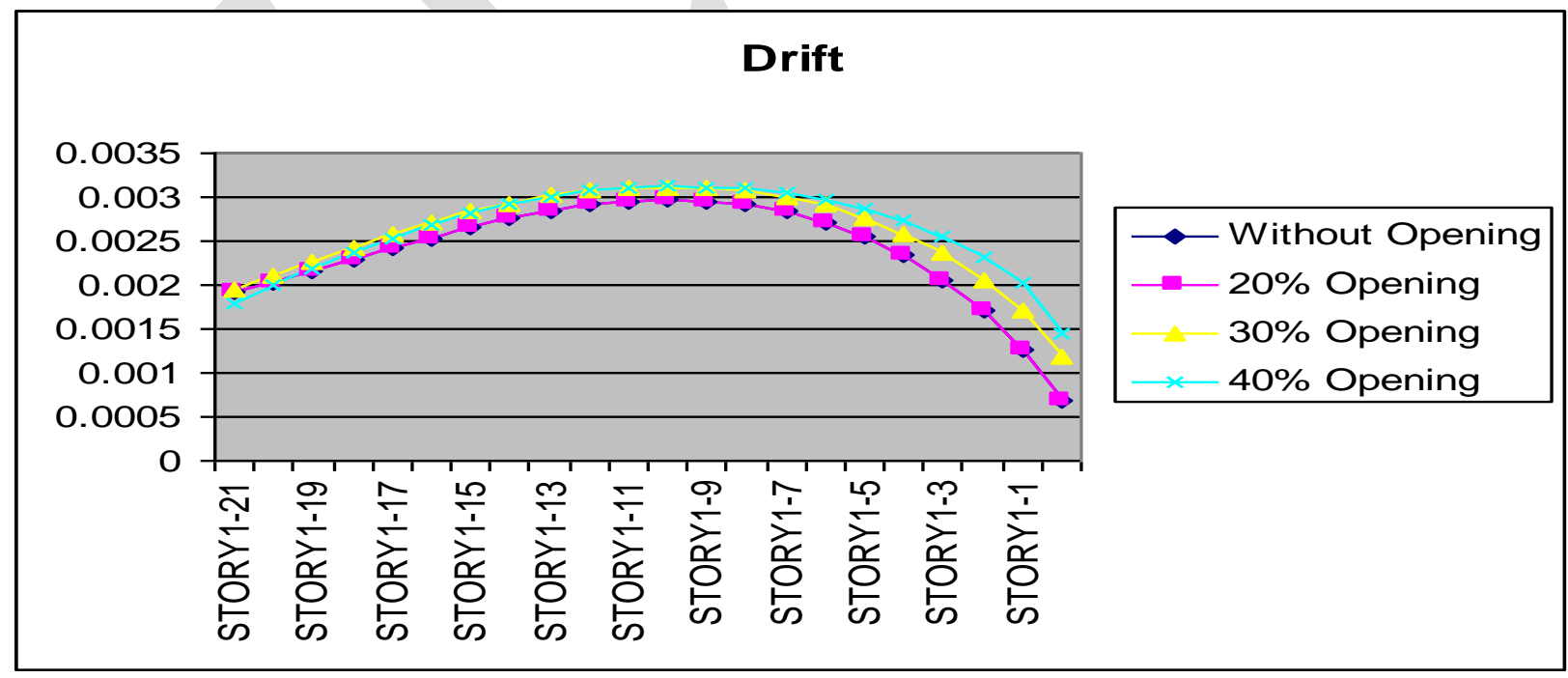

For Load Combinations $1.2(D . L+L . L+E Q Y N X)$ following are the values of the drift 
DOI : https://dx.doi.org/10.26808/rs.ed.i8v5.03

International Journal of Emerging Trends in Engineering and Development

Issue 8, Vol.5 (Aug-Sep 2018)

Available online on http://www.rspublication.com/ijeted/ijeted_index.htm

ISSN 2249-6149

\begin{tabular}{|c|c|c|c|c|c|c|c|}
\hline \multicolumn{8}{|c|}{ TABLE 6: Without Shear Wall } \\
\hline Story & Item & Load & Point & $X$ & $\mathrm{Y}$ & $\mathrm{Z}$ & DriftX \\
\hline STORY20 & Max Drift X & DCON25 & 8 & 28.94 & 26.845 & 61 & 0.000245 \\
\hline STORY19 & Max Drift X & DCON25 & 8 & 28.94 & 26.845 & 58 & 0.000268 \\
\hline STORY18 & Max Drift X & DCON25 & 8 & 28.94 & 26.845 & 55 & 0.00029 \\
\hline STORY17 & Max Drift X & DCON25 & 8 & 28.94 & 26.845 & 52 & 0.000311 \\
\hline STORY16 & Max Drift X & DCON25 & 8 & 28.94 & 26.845 & 49 & 0.000328 \\
\hline STORY15 & Max Drift X & DCON25 & 8 & 28.94 & 26.845 & 46 & 0.000343 \\
\hline STORY14 & Max Drift X & DCON25 & 8 & 28.94 & 26.845 & 43 & 0.000356 \\
\hline STORY13 & Max Drift X & DCON25 & 8 & 28.94 & 26.845 & 40 & 0.000365 \\
\hline STORY12 & Max Drift X & DCON25 & 8 & 28.94 & 26.845 & 37 & 0.000371 \\
\hline STORY11 & Max Drift X & DCON25 & 8 & 28.94 & 26.845 & 34 & 0.000374 \\
\hline STORY10 & Max Drift X & DCON25 & 8 & 28.94 & 26.845 & 31 & 0.000373 \\
\hline STORY9 & Max Drift X & DCON25 & 8 & 28.94 & 26.845 & 28 & 0.00037 \\
\hline STORY8 & Max Drift X & DCON25 & 8 & 28.94 & 26.845 & 25 & 0.000363 \\
\hline STORY7 & Max Drift X & DCON25 & 8 & 28.94 & 26.845 & 22 & 0.000353 \\
\hline STORY6 & Max Drift X & DCON25 & 8 & 28.94 & 26.845 & 19 & 0.000339 \\
\hline STORY5 & Max Drift X & DCON25 & 65 & 28.94 & 0 & 16 & 0.000328 \\
\hline STORY4 & Max Drift X & DCON25 & 65 & 28.94 & 0 & 13 & 0.000328 \\
\hline STORY3 & Max Drift X & DCON25 & 65 & 28.94 & 0 & 10 & 0.000332 \\
\hline STORY2 & Max Drift X & DCON25 & 65 & 28.94 & 0 & 7 & 0.000375 \\
\hline STORY1 & Max Drift X & DCON25 & 65 & 28.94 & 0 & 4 & 0.000739 \\
\hline
\end{tabular}


DOI : https://dx.doi.org/10.26808/rs.ed.i8v5.03

International Journal of Emerging Trends in Engineering and Development

Issue 8, Vol.5 (Aug-Sep 2018) Available online on http://www.rspublication.com/ijeted/ijeted_index.htm

ISSN 2249-6149

TABLE 7: Shear Wall Without Opening

\begin{tabular}{|c|c|c|c|c|c|c|c|}
\hline Story & Item & Load & Point & $X$ & $\mathrm{Y}$ & $\mathrm{Z}$ & DriftX \\
\hline STORY20 & Max Drift X & DCON25 & 8 & 1139.37 & 1056.89 & 2401.574 & 0.000324 \\
\hline STORY19 & Max Drift X & DCON25 & 8 & 1139.37 & 1056.89 & 2283.464 & 0.000328 \\
\hline STORY18 & Max Drift X & DCON25 & 65 & 1139.37 & 0 & 2165.354 & 0.000334 \\
\hline STORY17 & Max Drift X & DCON25 & 65 & 1139.37 & 0 & 2047.244 & 0.000341 \\
\hline STORY16 & Max Drift X & DCON25 & 65 & 1139.37 & 0 & 1929.133 & 0.000347 \\
\hline STORY15 & Max Drift X & DCON25 & 65 & 1139.37 & 0 & 1811.023 & 0.000352 \\
\hline STORY14 & Max Drift X & DCON25 & 65 & 1139.37 & 0 & 1692.913 & 0.000356 \\
\hline STORY13 & Max Drift X & DCON25 & 65 & 1139.37 & 0 & 1574.803 & 0.000358 \\
\hline STORY12 & Max Drift X & DCON25 & 65 & 1139.37 & 0 & 1456.693 & 0.000357 \\
\hline STORY11 & Max Drift X & DCON25 & 65 & 1139.37 & 0 & 1338.582 & 0.000353 \\
\hline STORY10 & Max Drift X & DCON25 & 65 & 1139.37 & 0 & 1220.472 & 0.000346 \\
\hline STORY9 & Max Drift X & DCON25 & 65 & 1139.37 & 0 & 1102.362 & 0.000336 \\
\hline STORY8 & Max Drift X & DCON25 & 65 & 1139.37 & 0 & 984.252 & 0.000321 \\
\hline STORY7 & Max Drift X & DCON25 & 65 & 1139.37 & 0 & 866.142 & 0.000302 \\
\hline STORY6 & Max Drift X & DCON25 & 65 & 1139.37 & 0 & 748.031 & 0.000279 \\
\hline STORY5 & Max Drift X & DCON25 & 65 & 1139.37 & 0 & 629.921 & 0.000251 \\
\hline STORY4 & Max Drift X & DCON25 & 65 & 1139.37 & 0 & 511.811 & 0.000216 \\
\hline STORY3 & Max Drift X & DCON25 & 65 & 1139.37 & 0 & 393.701 & 0.000175 \\
\hline STORY2 & Max Drift X & DCON25 & 65 & 1139.37 & 0 & 275.591 & 0.000126 \\
\hline STORY1 & Max Drift X & DCON25 & 65 & 1139.37 & 0 & 157.48 & 0.000059 \\
\hline
\end{tabular}


DOI : https://dx.doi.org/10.26808/rs.ed.i8v5.03

International Journal of Emerging Trends in Engineering and Development

Issue 8, Vol.5 (Aug-Sep 2018) Available online on http://www.rspublication.com/ijeted/ijeted_index.htm

ISSN 2249-6149

TABLE 8: Shear Wall with Opening

\begin{tabular}{|c|c|c|c|c|c|c|c|}
\hline Story & Item & Load & Point & $X$ & Y & $\mathrm{Z}$ & DriftX \\
\hline STORY20 & Max Drift X & DCON25 & 14-Jul & 975 & 1056.89 & 2366.141 & 0.000305 \\
\hline STORY19 & Max Drift X & DCON25 & 10-Jul & 975 & 1056.89 & 2177.165 & 0.000307 \\
\hline STORY18 & Max Drift X & DCON25 & 4-Jul & 975 & 1056.89 & 2070.866 & 0.000312 \\
\hline STORY17 & Max Drift X & DCON25 & 11-Jul & 975 & 1056.89 & 1964.566 & 0.000316 \\
\hline STORY16 & Max Drift X & DCON25 & 12-Jul & 975 & 1056.89 & 1858.267 & 0.00032 \\
\hline STORY15 & Max Drift X & DCON25 & 13-Jul & 975 & 1056.89 & 1751.968 & 0.000321 \\
\hline STORY14 & Max Drift X & DCON25 & 7-Jul & 975 & 1056.89 & 1645.669 & 0.000321 \\
\hline STORY13 & Max Drift X & DCON25 & 14-Jul & 975 & 1056.89 & 1539.37 & 0.00032 \\
\hline STORY12 & Max Drift X & DCON25 & 15-Jul & 975 & 1056.89 & 1433.07 & 0.000316 \\
\hline STORY11 & Max Drift X & DCON25 & 574 & 1090.059 & 0 & 1338.582 & 0.000312 \\
\hline STORY10 & Max Drift X & DCON25 & 602 & 90.827 & 0 & 1220.472 & 0.000307 \\
\hline STORY9 & Max Drift X & DCON25 & 603 & 75.689 & 0 & 1102.362 & 0.000299 \\
\hline STORY8 & Max Drift X & DCON25 & 604 & 60.551 & 0 & 984.252 & 0.000287 \\
\hline STORY7 & Max Drift X & DCON25 & 604 & 60.551 & 0 & 866.142 & 0.000272 \\
\hline STORY6 & Max Drift X & DCON25 & 605 & 45.413 & 0 & 748.031 & 0.000252 \\
\hline STORY5 & Max Drift X & DCON25 & 605 & 45.413 & 0 & 629.921 & 0.000227 \\
\hline STORY4 & Max Drift X & DCON25 & 605 & 45.413 & 0 & 511.811 & 0.000197 \\
\hline STORY3 & Max Drift X & DCON25 & 606 & 30.276 & 0 & 393.701 & 0.00016 \\
\hline STORY2 & Max Drift X & DCON25 & 576 & 1057.185 & 0 & 275.591 & 0.000116 \\
\hline STORY1 & Max Drift X & DCON25 & 604 & 60.551 & 0 & 157.48 & 0.000053 \\
\hline
\end{tabular}


DOI : https://dx.doi.org/10.26808/rs.ed.i8v5.03

International Journal of Emerging Trends in Engineering and Development

Issue 8, Vol.5 (Aug-Sep 2018) Available online on http://www.rspublication.com/ijeted/ijeted_index.htm

ISSN 2249-6149

The following are the values of the Center Of Rigidity and Center Of Mass

TABLE 9: With Out Shear Wall

\begin{tabular}{|c|c|c|c|c|c|c|c|c|}
\hline Story & MassX & MassY & $\mathrm{XCM}$ & YCM & $\mathrm{XCCM}$ & YCCM & XCR & YCR \\
\hline STORY20 & 1730.674 & 1730.674 & 14.683 & 13.481 & 14.683 & 13.481 & 14.684 & 15.016 \\
\hline STORY19 & 1960.744 & 1960.744 & 14.672 & 13.526 & 14.677 & 13.505 & 14.676 & 15.075 \\
\hline STORY18 & 1960.744 & 1960.744 & 14.672 & 13.526 & 14.675 & 13.512 & 14.668 & 15.136 \\
\hline STORY17 & 1960.744 & 1960.744 & 14.672 & 13.526 & 14.674 & 13.516 & 14.66 & 15.192 \\
\hline STORY16 & 1960.744 & 1960.744 & 14.672 & 13.526 & 14.674 & 13.518 & 14.652 & 15.245 \\
\hline STORY15 & 1960.744 & 1960.744 & 14.672 & 13.526 & 14.673 & 13.519 & 14.645 & 15.299 \\
\hline STORY14 & 1960.744 & 1960.744 & 14.672 & 13.526 & 14.673 & 13.52 & 14.637 & 15.355 \\
\hline STORY13 & 1960.744 & 1960.744 & 14.672 & 13.526 & 14.673 & 13.521 & 14.63 & 15.416 \\
\hline STORY12 & 1960.744 & 1960.744 & 14.672 & 13.526 & 14.673 & 13.522 & 14.622 & 15.483 \\
\hline STORY11 & 1960.744 & 1960.744 & 14.672 & 13.526 & 14.673 & 13.522 & 14.614 & 15.556 \\
\hline STORY10 & 1960.744 & 1960.744 & 14.672 & 13.526 & 14.672 & 13.522 & 14.606 & 15.637 \\
\hline STORY9 & 1960.744 & 1960.744 & 14.672 & 13.526 & 14.672 & 13.523 & 14.598 & 15.727 \\
\hline STORY8 & 1960.744 & 1960.744 & 14.672 & 13.526 & 14.672 & 13.523 & 14.59 & 15.825 \\
\hline STORY7 & 1960.744 & 1960.744 & 14.672 & 13.526 & 14.672 & 13.523 & 14.582 & 15.931 \\
\hline STORY6 & 1960.744 & 1960.744 & 14.672 & 13.526 & 14.672 & 13.523 & 14.573 & 16.045 \\
\hline STORY5 & 1960.744 & 1960.744 & 14.672 & 13.526 & 14.672 & 13.523 & 14.565 & 16.164 \\
\hline STORY4 & 1960.744 & 1960.744 & 14.672 & 13.526 & 14.672 & 13.524 & 14.556 & 16.282 \\
\hline STORY3 & 1960.744 & 1960.744 & 14.672 & 13.526 & 14.672 & 13.524 & 14.548 & 16.388 \\
\hline STORY2 & 1960.744 & 1960.744 & 14.672 & 13.526 & 14.672 & 13.524 & 14.542 & 16.451 \\
\hline STORY1 & 2034.611 & 2034.611 & 14.668 & 13.539 & 14.672 & 13.525 & 14.543 & 16.397 \\
\hline
\end{tabular}


DOI : https://dx.doi.org/10.26808/rs.ed.i8v5.03 International Journal of Emerging Trends in Engineering and Development Issue 8, Vol.5 (Aug-Sep 2018) Available online on http://www.rspublication.com/ijeted/ijeted_index.htm

ISSN 2249-6149

\section{TABLE 10: Shear Wall With Out Opening}

\begin{tabular}{|c|c|c|c|c|c|c|c|c|}
\hline Story & MassX & MassY & $\mathrm{XCM}$ & YCM & XCCM & YCCM & XCR & YCR \\
\hline STORY20 & 1853.364 & 1853.364 & 14.686 & 13.417 & 14.686 & 13.417 & 14.742 & 14.466 \\
\hline STORY19 & 2206.123 & 2206.123 & 14.678 & 13.413 & 14.682 & 13.415 & 14.742 & 14.47 \\
\hline STORY18 & 2206.123 & 2206.123 & 14.678 & 13.413 & 14.681 & 13.414 & 14.743 & 14.476 \\
\hline STORY17 & 2206.123 & 2206.123 & 14.678 & 13.413 & 14.68 & 13.414 & 14.744 & 14.483 \\
\hline STORY16 & 2206.123 & 2206.123 & 14.678 & 13.413 & 14.68 & 13.414 & 14.745 & 14.49 \\
\hline STORY15 & 2206.123 & 2206.123 & 14.678 & 13.413 & 14.679 & 13.414 & 14.746 & 14.499 \\
\hline STORY14 & 2206.123 & 2206.123 & 14.678 & 13.413 & 14.679 & 13.414 & 14.748 & 14.508 \\
\hline STORY13 & 2206.123 & 2206.123 & 14.678 & 13.413 & 14.679 & 13.414 & 14.749 & 14.517 \\
\hline STORY12 & 2206.123 & 2206.123 & 14.678 & 13.413 & 14.679 & 13.414 & 14.751 & 14.526 \\
\hline STORY11 & 2206.123 & 2206.123 & 14.678 & 13.413 & 14.679 & 13.414 & 14.752 & 14.535 \\
\hline STORY10 & 2206.123 & 2206.123 & 14.678 & 13.413 & 14.679 & 13.414 & 14.754 & 14.542 \\
\hline STORY9 & 2206.123 & 2206.123 & 14.678 & 13.413 & 14.679 & 13.414 & 14.756 & 14.549 \\
\hline STORY8 & 2206.123 & 2206.123 & 14.678 & 13.413 & 14.679 & 13.414 & 14.759 & 14.553 \\
\hline STORY7 & 2206.123 & 2206.123 & 14.678 & 13.413 & 14.679 & 13.414 & 14.76 & 14.554 \\
\hline STORY6 & 2206.123 & 2206.123 & 14.678 & 13.413 & 14.679 & 13.414 & 14.761 & 14.551 \\
\hline STORY5 & 2206.123 & 2206.123 & 14.678 & 13.413 & 14.679 & 13.414 & 14.76 & 14.54 \\
\hline STORY4 & 2206.123 & 2206.123 & 14.678 & 13.413 & 14.679 & 13.414 & 14.755 & 14.517 \\
\hline STORY3 & 2206.123 & 2206.123 & 14.678 & 13.413 & 14.678 & 13.414 & 14.741 & 14.475 \\
\hline STORY2 & 2206.123 & 2206.123 & 14.678 & 13.413 & 14.678 & 13.414 & 14.711 & 14.399 \\
\hline STORY1 & 2320.887 & 2320.887 & 14.676 & 13.413 & 14.678 & 13.414 & 14.651 & 14.272 \\
\hline
\end{tabular}


DOI : https://dx.doi.org/10.26808/rs.ed.i8v5.03

International Journal of Emerging Trends in Engineering and Development

Issue 8, Vol.5 (Aug-Sep 2018) Available online on http://www.rspublication.com/ijeted/ijeted_index.htm

ISSN 2249-6149

TABLE 11: Shear Wall With Opening

\begin{tabular}{|c|c|c|c|c|c|c|c|c|}
\hline Story & MassX & MassY & $\mathrm{XCM}$ & YCM & $\mathrm{XCCM}$ & YCCM & $\mathrm{XCR}$ & YCR \\
\hline STORY20 & 1743.045 & 1743.045 & 14.683 & 13.475 & 14.683 & 13.475 & 14.734 & 14.494 \\
\hline STORY19 & 1985.384 & 1985.384 & 14.672 & 13.514 & 14.677 & 13.496 & 14.734 & 14.5 \\
\hline STORY18 & 1985.384 & 1985.384 & 14.672 & 13.514 & 14.676 & 13.502 & 14.735 & 14.507 \\
\hline STORY17 & 1985.384 & 1985.384 & 14.672 & 13.514 & 14.675 & 13.505 & 14.735 & 14.516 \\
\hline STORY16 & 1985.384 & 1985.384 & 14.672 & 13.514 & 14.674 & 13.507 & 14.736 & 14.526 \\
\hline STORY15 & 1985.384 & 1985.384 & 14.672 & 13.514 & 14.674 & 13.508 & 14.736 & 14.536 \\
\hline STORY14 & 1985.384 & 1985.384 & 14.672 & 13.514 & 14.674 & 13.509 & 14.737 & 14.547 \\
\hline STORY13 & 1985.384 & 1985.384 & 14.672 & 13.514 & 14.673 & 13.51 & 14.737 & 14.558 \\
\hline STORY12 & 1985.384 & 1985.384 & 14.672 & 13.514 & 14.673 & 13.51 & 14.738 & 14.57 \\
\hline STORY11 & 1985.384 & 1985.384 & 14.672 & 13.514 & 14.673 & 13.511 & 14.739 & 14.581 \\
\hline STORY10 & 1985.384 & 1985.384 & 14.672 & 13.514 & 14.673 & 13.511 & 14.739 & 14.592 \\
\hline STORY9 & 1985.384 & 1985.384 & 14.672 & 13.514 & 14.673 & 13.511 & 14.74 & 14.602 \\
\hline STORY8 & 1985.384 & 1985.384 & 14.672 & 13.514 & 14.673 & 13.511 & 14.741 & 14.61 \\
\hline STORY7 & 1985.384 & 1985.384 & 14.672 & 13.514 & 14.673 & 13.512 & 14.741 & 14.616 \\
\hline STORY6 & 1985.384 & 1985.384 & 14.672 & 13.514 & 14.673 & 13.512 & 14.74 & 14.618 \\
\hline STORY5 & 1985.384 & 1985.384 & 14.672 & 13.514 & 14.673 & 13.512 & 14.736 & 14.612 \\
\hline STORY4 & 1985.384 & 1985.384 & 14.672 & 13.514 & 14.673 & 13.512 & 14.729 & 14.592 \\
\hline STORY3 & 1985.384 & 1985.384 & 14.672 & 13.514 & 14.673 & 13.512 & 14.714 & 14.549 \\
\hline STORY2 & 1985.384 & 1985.384 & 14.672 & 13.514 & 14.673 & 13.512 & 14.692 & 14.466 \\
\hline STORY1 & 2063.341 & 2063.341 & 14.669 & 13.526 & 14.673 & 13.513 & 14.658 & 14.342 \\
\hline
\end{tabular}


DOI : https://dx.doi.org/10.26808/rs.ed.i8v5.03

International Journal of Emerging Trends in Engineering and Development

Issue 8, Vol.5 (Aug-Sep 2018) Available online on http://www.rspublication.com/ijeted/ijeted_index.htm

ISSN 2249-6149

The following are the values of the Model Mass Contributions

TABLE 12: With Out Shear Wall

\begin{tabular}{|c|c|c|c|c|c|c|}
\hline Mode & Period & UX & UY & UZ & SumUX & SumUY \\
\hline 1 & 2.813121 & 13.2781 & 0.005 & 0 & 13.2781 & 0.005 \\
\hline 2 & 2.326692 & 0.1383 & 75.9988 & 0 & 13.4164 & 76.0038 \\
\hline 3 & 2.204959 & 62.6667 & 0.1973 & 0 & 76.0831 & 76.2011 \\
\hline 4 & 0.923186 & 1.6732 & 0.0004 & 0 & 77.7563 & 76.2015 \\
\hline 5 & 0.704249 & 0.0047 & 12.6577 & 0 & 77.761 & 88.8591 \\
\hline 6 & 0.653931 & 12.2647 & 0.0051 & 0 & 90.0257 & 88.8642 \\
\hline 7 & 0.505037 & 0.5488 & 0.0004 & 0 & 90.5745 & 88.8647 \\
\hline 8 & 0.364535 & 0 & 4.5899 & 0 & 90.5745 & 93.4545 \\
\hline 9 & 0.346039 & 0.2477 & 0.0017 & 0 & 90.8223 & 93.4563 \\
\hline 10 & 0.332068 & 4.3894 & 0 & 0 & 95.2117 & 93.4563 \\
\hline 11 & 0.256092 & 0.0234 & 0 & 0 & 95.235 & 93.4563 \\
\hline 12 & 0.232316 & 0 & 2.4896 & 0 & 95.235 & 95.9459 \\
\hline
\end{tabular}

TABLE 13: Shear Wall Without Opening

\begin{tabular}{|c|c|c|c|c|c|c|}
\hline Mode & Period & UX & UY & UZ & SumUX & SumUY \\
\hline 1 & 2.008698 & 65.6283 & 0.5892 & 0 & 65.6283 & 0.5892 \\
\hline 2 & 1.895758 & 0.6233 & 72.4332 & 0 & 66.2516 & 73.0224 \\
\hline 3 & 1.75645 & 6.369 & 0.0291 & 0 & 72.6205 & 73.0515 \\
\hline 4 & 0.554075 & 13.8419 & 0.1119 & 0 & 86.4625 & 73.1634 \\
\hline 5 & 0.53466 & 0.1138 & 13.5512 & 0 & 86.5763 & 86.7146 \\
\hline 6 & 0.453777 & 0.5506 & 0.0041 & 0 & 87.1268 & 86.7187 \\
\hline 7 & 0.263488 & 5.5609 & 0.0142 & 0 & 92.6877 & 86.7329 \\
\hline 8 & 0.256385 & 0.0148 & 5.2883 & 0 & 92.7025 & 92.0211 \\
\hline 9 & 0.203708 & 0.1221 & 0.0013 & 0 & 92.8246 & 92.0224 \\
\hline 10 & 0.160475 & 2.9063 & 0.0006 & 0 & 95.7309 & 92.023 \\
\hline 11 & 0.153942 & 0.0006 & 2.9382 & 0 & 95.7315 & 94.9612 \\
\hline 12 & 0.119972 & 0.0556 & 0.0006 & 0 & 95.7871 & 95.2659 \\
\hline
\end{tabular}


DOI : https://dx.doi.org/10.26808/rs.ed.i8v5.03 International Journal of Emerging Trends in Engineering and Development Available online on http://www.rspublication.com/ijeted/ijeted_index.htm

TABLE 14: Shear Wall With Opening

\begin{tabular}{|c|c|c|c|c|c|c|}
\hline Mode & Period & UX & UY & UZ & SumUX & SumUY \\
\hline 1 & 2.037052 & 64.4361 & 0.3771 & 0 & 64.4361 & 0.3771 \\
\hline 2 & 1.933803 & 0.412 & 72.9029 & 0 & 64.8481 & 73.2801 \\
\hline 3 & 1.794027 & 7.6779 & 0.033 & 0 & 72.5261 & 73.3131 \\
\hline 4 & 0.564611 & 13.6862 & 0.1278 & 0 & 86.2123 & 73.4409 \\
\hline 5 & 0.551598 & 0.1336 & 13.4243 & 0 & 86.3459 & 86.8653 \\
\hline 6 & 0.47132 & 0.7877 & 0.003 & 0 & 87.1336 & 86.8682 \\
\hline 7 & 0.269714 & 1.8097 & 3.4556 & 0 & 88.9433 & 90.3238 \\
\hline 8 & 0.269188 & 3.7129 & 1.6846 & 0 & 92.6563 & 92.0084 \\
\hline 9 & 0.216015 & 0.2079 & 0.0007 & 0 & 92.8642 & 92.0091 \\
\hline 10 & 0.165567 & 0.005 & 2.7647 & 0 & 92.8692 & 94.7738 \\
\hline 11 & 0.164697 & 2.8754 & 0.0048 & 0 & 95.7446 & 94.7786 \\
\hline 12 & 0.129794 & 0.0964 & 0.0002 & 0 & 95.8409 & 95.2785 \\
\hline
\end{tabular}

Shear Force, Bend Motion and Torsion

TABLE 15: Without Shear Wall

\begin{tabular}{|c|c|c|c|c|c|c|c|c|c|}
\hline Story & Column & Load & Loc & $\mathrm{P}$ & $\mathrm{V} 2$ & $\mathrm{~V} 3$ & $\mathrm{~T}$ & $\mathrm{M} 2$ & $\mathrm{M} 3$ \\
\hline STORY20 & $\mathrm{C} 23$ & DCON25 & 0 & -297.11 & 14.82 & 87.26 & -2.348 & 111.457 & 19.706 \\
\hline STORY19 & $\mathrm{C} 23$ & DCON25 & 0 & -571.87 & 12.79 & 77.45 & -2.725 & 111.017 & 18.904 \\
\hline STORY18 & $\mathrm{C} 23$ & DCON25 & 0 & -847.37 & 14.1 & 93.04 & -3.112 & 131.185 & 20.77 \\
\hline STORY17 & $\mathrm{C} 23$ & DCON25 & 0 & -1118.36 & 15.15 & 106.63 & -3.471 & 151.669 & 22.405 \\
\hline STORY16 & $\mathrm{C} 23$ & DCON25 & 0 & -1385.85 & 16.12 & 120.7 & -3.793 & 173.085 & 23.95 \\
\hline STORY15 & $\mathrm{C} 23$ & DCON25 & 0 & -1650.26 & 17.01 & 134.05 & -4.074 & 193.812 & 25.376 \\
\hline STORY14 & $\mathrm{C} 23$ & DCON25 & 0 & -1912.27 & 17.78 & 146.33 & -4.313 & 213.132 & 26.625 \\
\hline STORY13 & $\mathrm{C} 23$ & DCON25 & 0 & -2172.61 & 18.41 & 157.24 & -4.509 & 230.534 & 27.659 \\
\hline STORY12 & $\mathrm{C} 23$ & DCON25 & 0 & -2431.99 & 18.87 & 166.61 & -4.663 & 245.694 & 28.442 \\
\hline STORY11 & $\mathrm{C} 23$ & DCON25 & 0 & -2691.11 & 19.14 & 174.29 & -4.775 & 258.387 & 28.935 \\
\hline STORY10 & $\mathrm{C} 23$ & DCON25 & 0 & -2950.64 & 19.19 & 180.19 & -4.845 & 268.437 & 29.103 \\
\hline STORY9 & $\mathrm{C} 23$ & DCON25 & 0 & -3211.27 & 19 & 184.15 & -4.875 & 275.668 & 28.907 \\
\hline STORY8 & $\mathrm{C} 23$ & DCON25 & 0 & -3473.68 & 18.54 & 186.02 & -4.866 & 279.867 & 28.305 \\
\hline STORY7 & $\mathrm{C} 23$ & DCON25 & 0 & -3738.66 & 17.78 & 185.52 & -4.817 & 280.72 & 27.256 \\
\hline STORY6 & $\mathrm{C} 23$ & DCON25 & 0 & -4007.14 & 16.69 & 182.25 & -4.731 & 277.768 & 25.7 \\
\hline STORY5 & $\mathrm{C} 23$ & DCON25 & 0 & -4280.35 & 15.24 & 175.48 & -4.609 & 270.134 & 23.609 \\
\hline STORY4 & C23 & DCON25 & 0 & -4560 & 13.31 & 164.49 & -4.465 & 257.474 & 20.749 \\
\hline STORY3 & C23 & DCON25 & 0 & -4848.9 & 11.07 & 144.99 & -4.351 & 232.329 & 17.579 \\
\hline STORY2 & C23 & DCON25 & 0 & -5150.04 & 6.97 & 134.27 & -4.621 & 241.03 & 9.722 \\
\hline STORY1 & C23 & DCON25 & 0 & -5495.36 & 3.77 & -5.24 & 0 & 0 & 0 \\
\hline
\end{tabular}


DOI : https://dx.doi.org/10.26808/rs.ed.i8v5.03

International Journal of Emerging Trends in Engineering and Development

Issue 8, Vol.5 (Aug-Sep 2018) Available online on http://www.rspublication.com/ijeted/ijeted_index.htm

ISSN 2249-6149

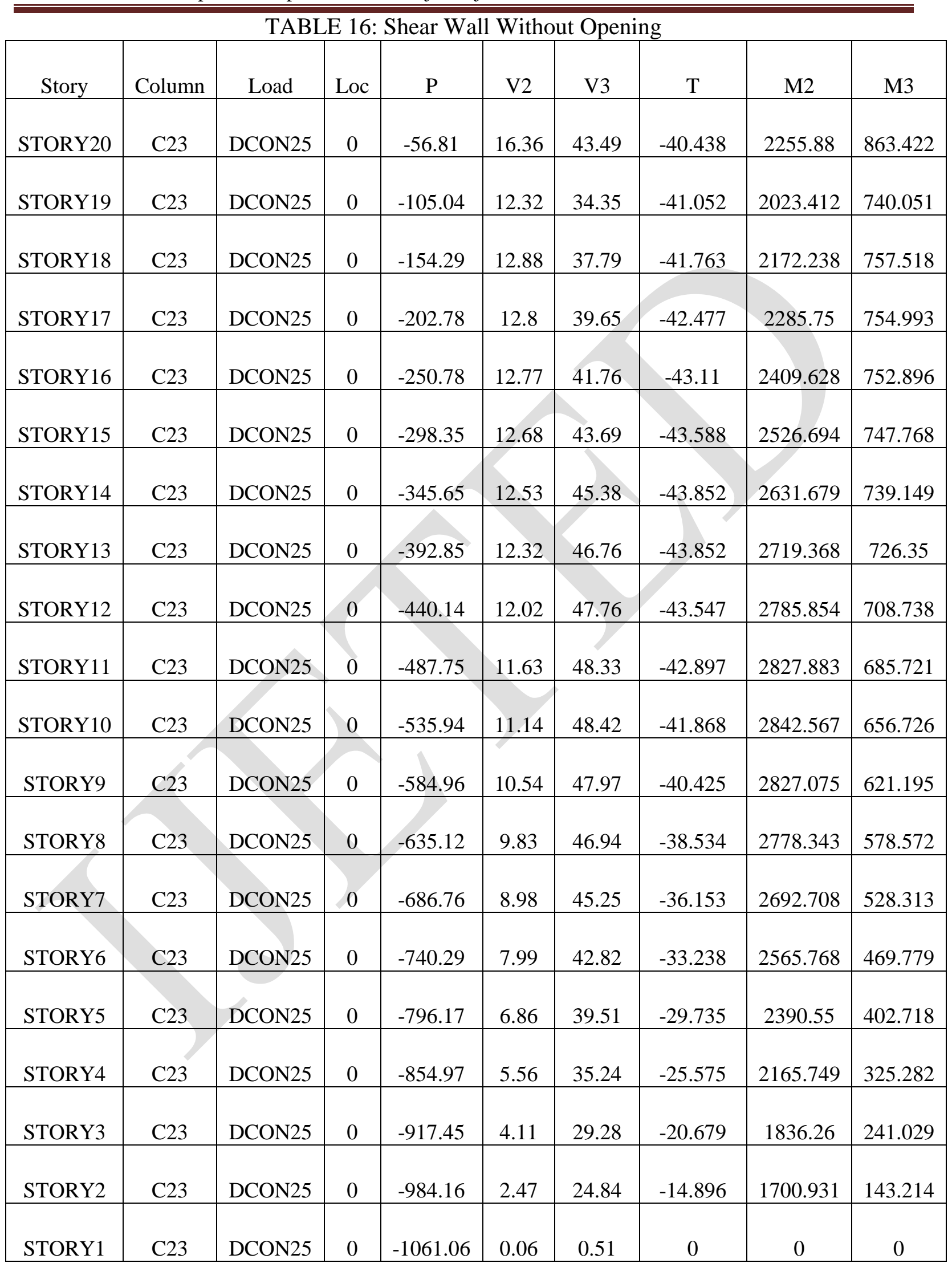


DOI : https://dx.doi.org/10.26808/rs.ed.i8v5.03

International Journal of Emerging Trends in Engineering and Development

Issue 8, Vol.5 (Aug-Sep 2018) Available online on http://www.rspublication.com/ijeted/ijeted_index.htm

ISSN 2249-6149

\section{TABLE 17: Shear Wall With Opening}

\begin{tabular}{|c|c|c|c|c|c|c|c|c|c|}
\hline Story & Column & Load & Loc & $\mathrm{P}$ & $\mathrm{V} 2$ & V3 & $\mathrm{T}$ & M2 & M3 \\
\hline STORY20 & $\mathrm{C} 23$ & DCON25 & 0 & -58.05 & 14.78 & 41.42 & -35.714 & 2142.233 & 780.131 \\
\hline STORY19 & $\mathrm{C} 23$ & DCON25 & 0 & -107.72 & 11.16 & 33.3 & -36.409 & 1952.634 & 669.768 \\
\hline STORY18 & $\mathrm{C} 23$ & DCON25 & 0 & -158.24 & 11.67 & 36.78 & -37.163 & 2109.614 & 686.42 \\
\hline STORY17 & $\mathrm{C} 23$ & DCON25 & 0 & -207.93 & 11.62 & 38.82 & -37.922 & 2233.694 & 684.96 \\
\hline STORY16 & $\mathrm{C} 23$ & DCON25 & 0 & -257.08 & 11.6 & 41.05 & -38.612 & 2365.897 & 683.9 \\
\hline STORY15 & $\mathrm{C} 23$ & DCON25 & 0 & -305.76 & 11.53 & 43.1 & -39.16 & 2490.336 & 680.069 \\
\hline STORY14 & $\mathrm{C} 23$ & DCON25 & 0 & -354.13 & 11.41 & 44.9 & -39.513 & 2601.966 & 673.043 \\
\hline STORY13 & $\mathrm{C} 23$ & DCON25 & 0 & -402.36 & 11.23 & 46.38 & -39.625 & 2695.856 & 662.172 \\
\hline STORY12 & $\mathrm{C} 23$ & DCON25 & 0 & -450.65 & 10.97 & 47.47 & -39.455 & 2768.288 & 646.864 \\
\hline STORY11 & $\mathrm{C} 23$ & DCON25 & 0 & -499.22 & 10.62 & 48.14 & -38.968 & 2816.161 & 626.554 \\
\hline STORY10 & $\mathrm{C} 23$ & DCON25 & 0 & -548.3 & 10.19 & 48.32 & -38.131 & 2836.724 & 600.697 \\
\hline STORY9 & $\mathrm{C} 23$ & DCON25 & 0 & -598.15 & 9.65 & 47.98 & -36.911 & 2827.286 & 568.756 \\
\hline STORY8 & $\mathrm{C} 23$ & DCON25 & 0 & -649.07 & 9 & 47.06 & -35.274 & 2784.95 & 530.192 \\
\hline STORY7 & $\mathrm{C} 23$ & DCON25 & 0 & -701.39 & 8.23 & 45.5 & -33.18 & 2706.281 & 484.478 \\
\hline STORY6 & $\mathrm{C} 23$ & DCON25 & 0 & -755.47 & 7.32 & 43.2 & -30.583 & 2587.163 & 430.986 \\
\hline STORY5 & $\mathrm{C} 23$ & DCON25 & 0 & -811.76 & 6.28 & 40.06 & -27.429 & 2421.141 & 369.474 \\
\hline STORY4 & $\mathrm{C} 23$ & DCON25 & 0 & -870.81 & 5.08 & 35.99 & -23.645 & 2207.253 & 298.057 \\
\hline STORY3 & $\mathrm{C} 23$ & DCON25 & 0 & -933.32 & 3.75 & 30.28 & -19.15 & 1891.258 & 220.525 \\
\hline STORY2 & $\mathrm{C} 23$ & DCON25 & 0 & -999.81 & 2.22 & 26.45 & -13.773 & 1811.38 & 128.735 \\
\hline STORY1 & $\mathrm{C} 23$ & DCON25 & 0 & -1076.58 & 0.03 & 0.32 & 0 & 0 & 0 \\
\hline
\end{tabular}




\section{GRAPHS}

Comparison of Story Drift for C23

1) Without Shear Wall

2) Shear Wall Without Opening

3) Shear Wall With Opening

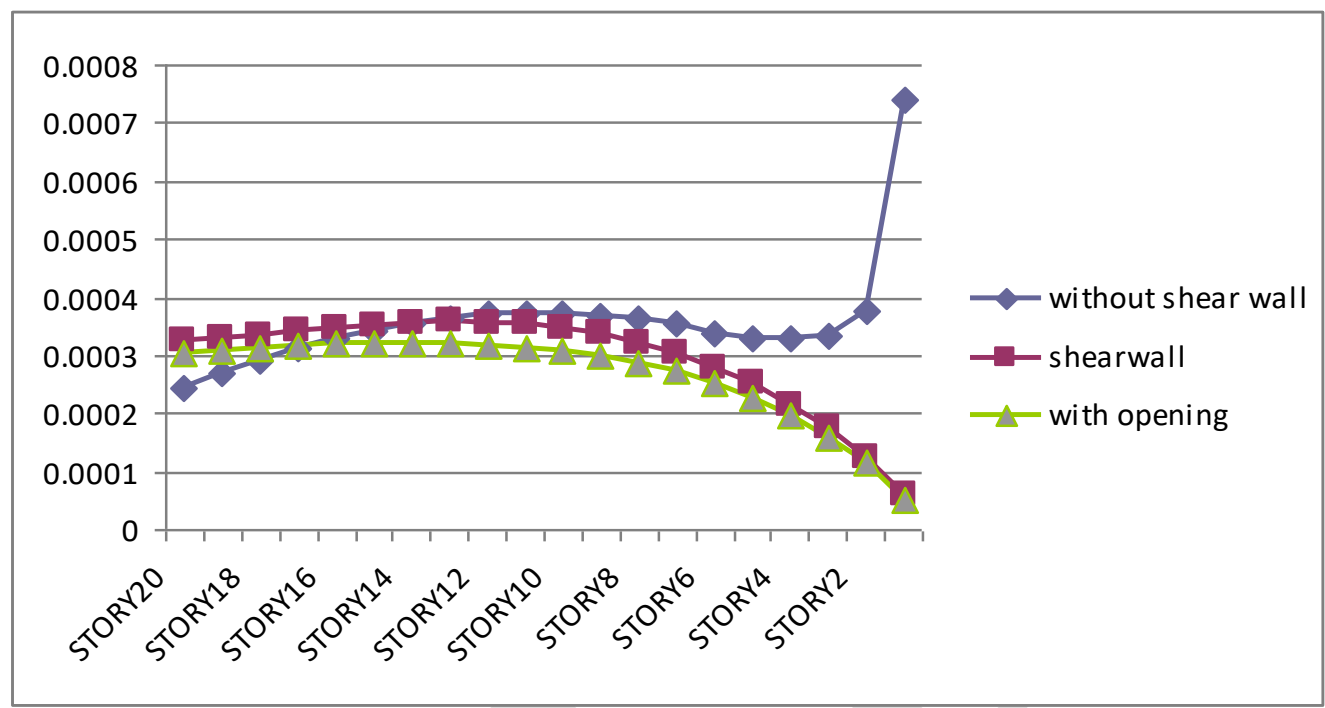

Graph 4: Load Combinations 1.2 (D.L + L.L + EQYNX)

\section{Comparison of Shear Force for C23}

4) Without Shear Wall

5) Shear Wall Without Opening

6) Shear Wall With Opening

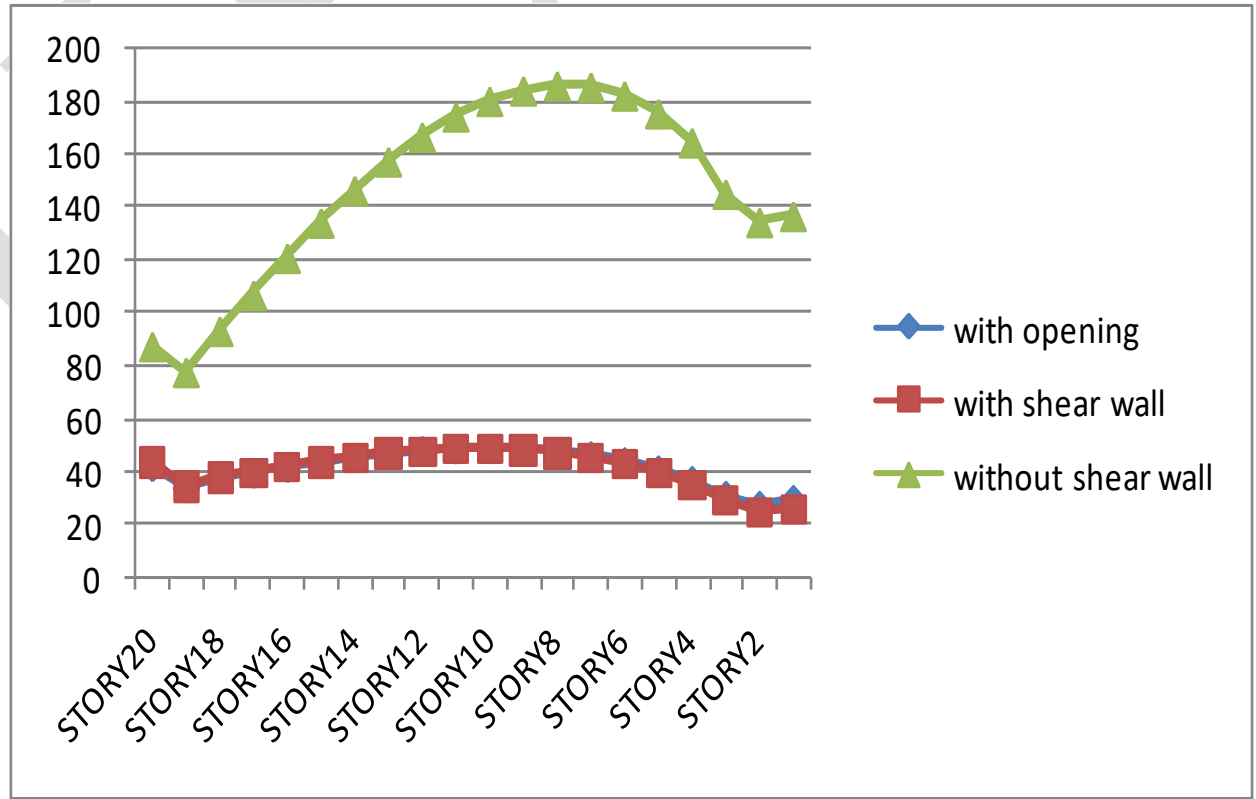

Graph 5: Load Combinations 1.2 (D.L + L.L + EQYNX) 


\section{Comparison of Torsion for $\mathrm{C} 23$}

7) Without Shear Wall

8) Shear Wall Without Opening

9) Shear Wall With Opening

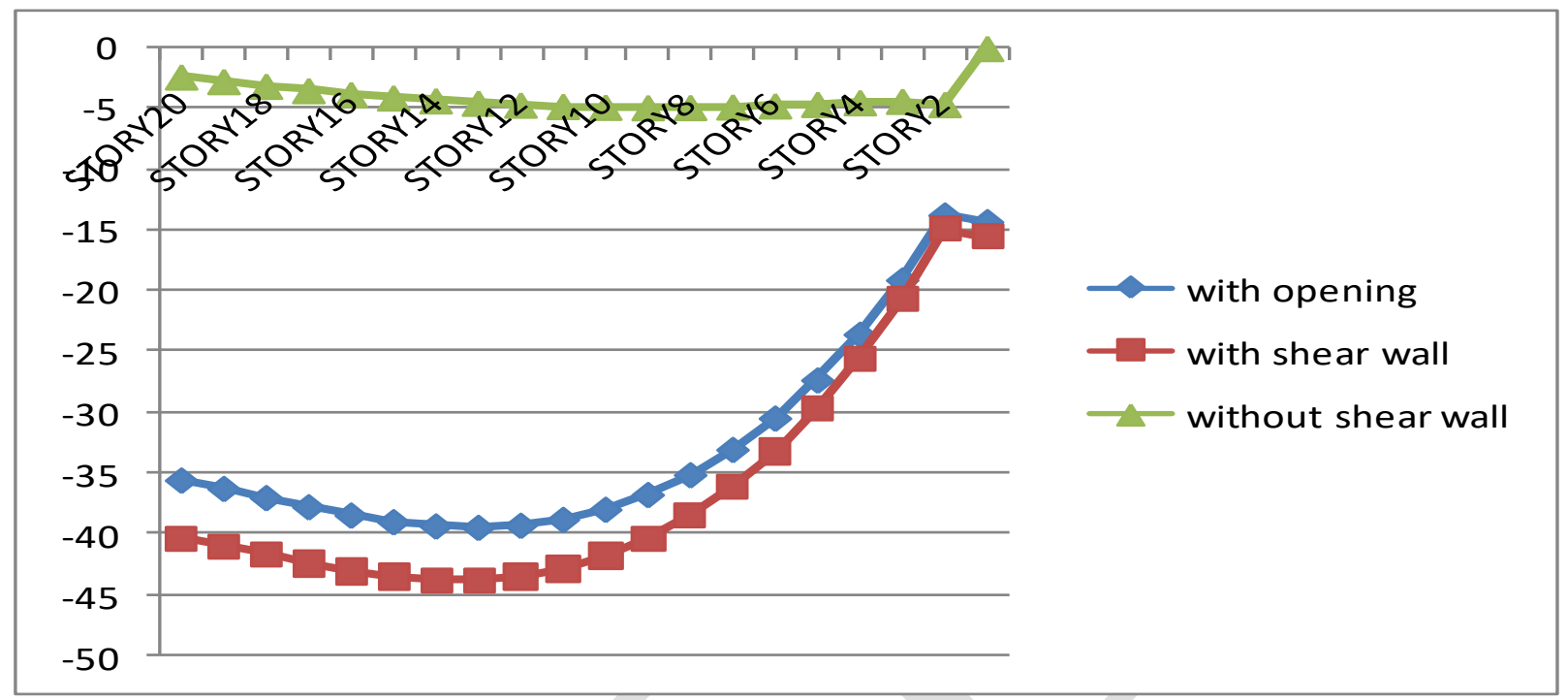

Graph 6: Load Combinations 1.2 (D.L + L.L + EQYNX)

\section{Comparison of Bending Moment for C23}

10)Without Shear Wall

11) Shear Wall Without Opening

12) Shear Wall With Opening

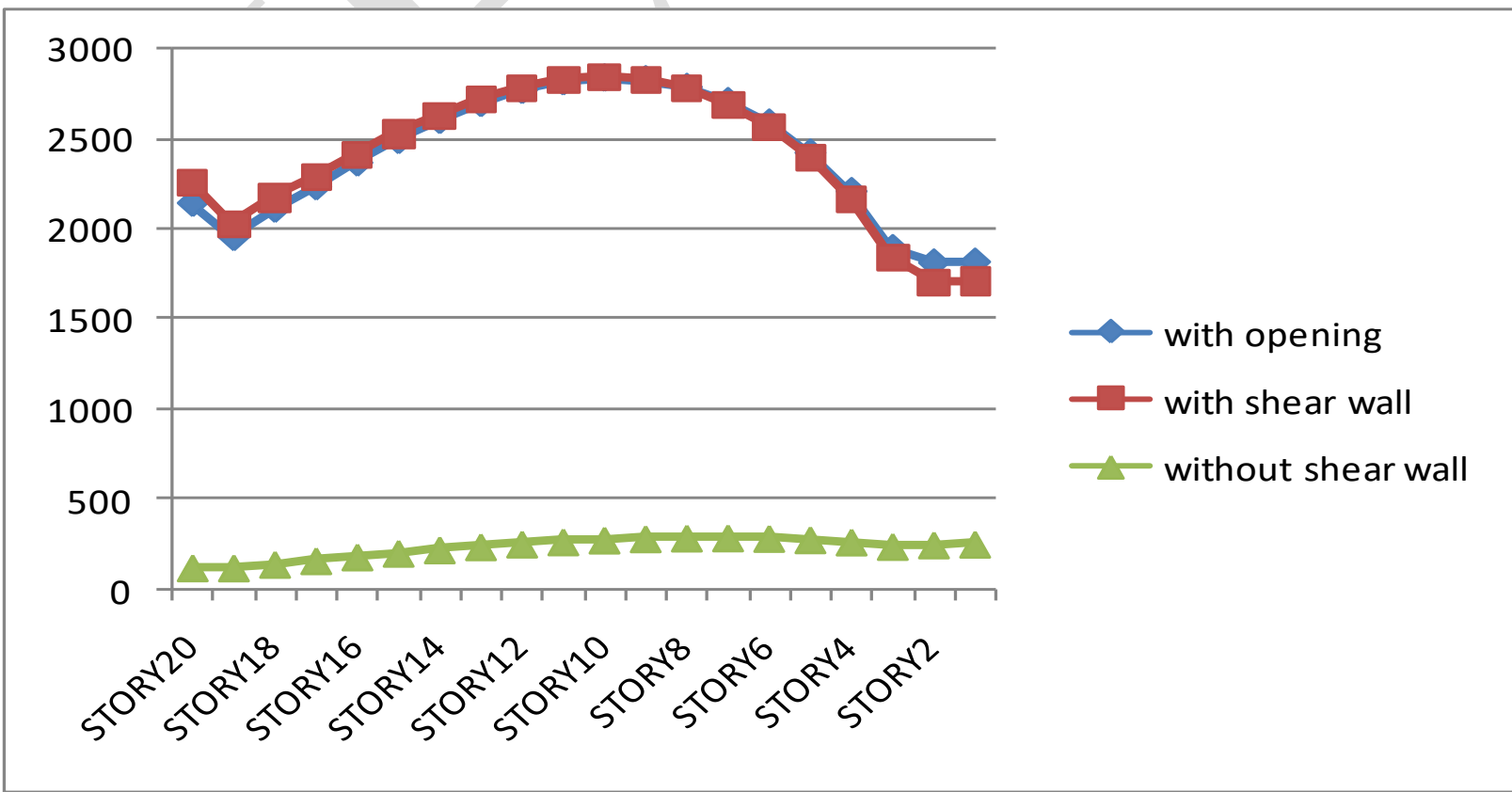

Graph 7: Load Combinations 1.2 (D.L + L.L + EQYNX) 
Check for Drift and Displacement

From IS 1893 (Part - I) - 2002

Allowable drift $=0.004 \mathrm{~h}=0.004 \times 3=0.012 \mathrm{~m}$

Allowable displacement $=\mathrm{H} / 500=0.122 \mathrm{~m}$

Case 1:- Shear Wall with Openings

For Load Combination EQXNY

Drift $=0.0021522 \mathrm{~m}$

Displacement $=0.11 \mathrm{~m}$

Case 2:- Shear Wall without Openings

For Load Combination EQXNY

Drift $=0.00213 \mathrm{~m}$

Displacement $=0.1 \mathrm{~m}$

Case 3:- Without Shear Wall

For Load Combination EQXNY

Drift $=0.0021522 \mathrm{~m}$

Displacement $=0.15 \mathrm{~m}$

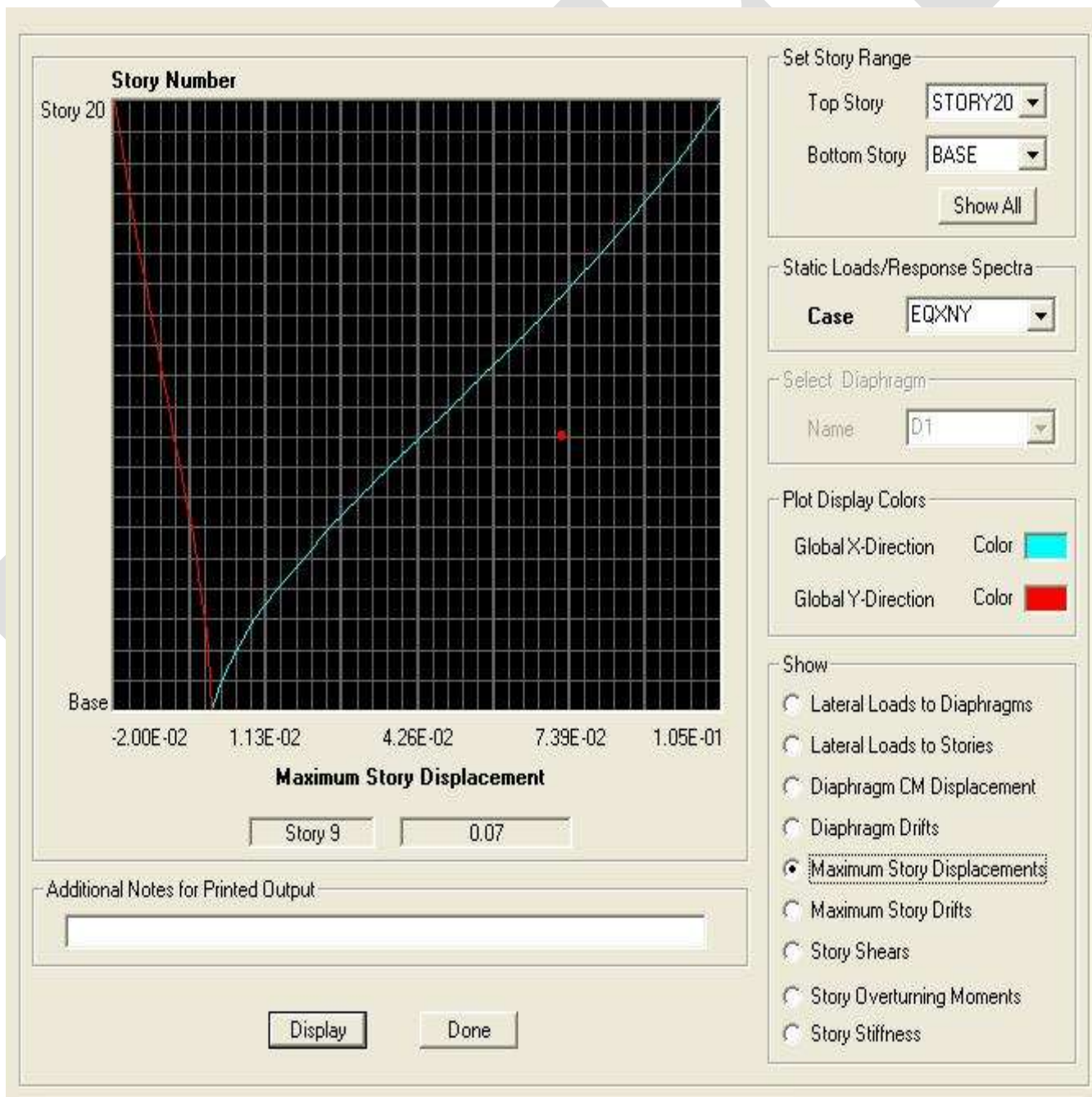

Graph 8: Displacement Shear Wall 
DOI : https://dx.doi.org/10.26808/rs.ed.i8v5.03

International Journal of Emerging Trends in Engineering and Development

Issue 8, Vol.5 (Aug-Sep 2018)

Available online on http://www.rspublication.com/ijeted/ijeted_index.htm

ISSN 2249-6149

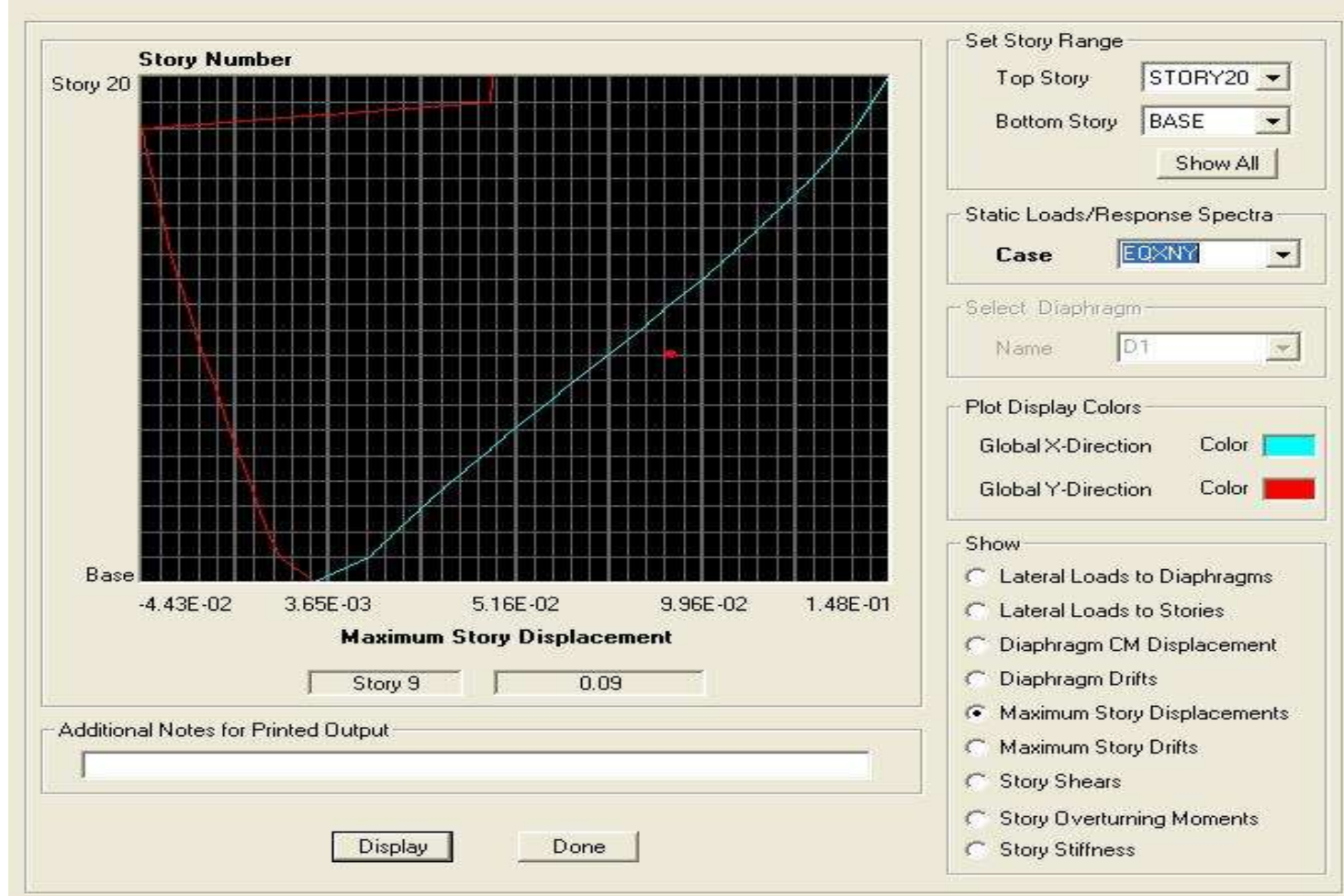

Graph 9: Displacement With Out Shear Wall

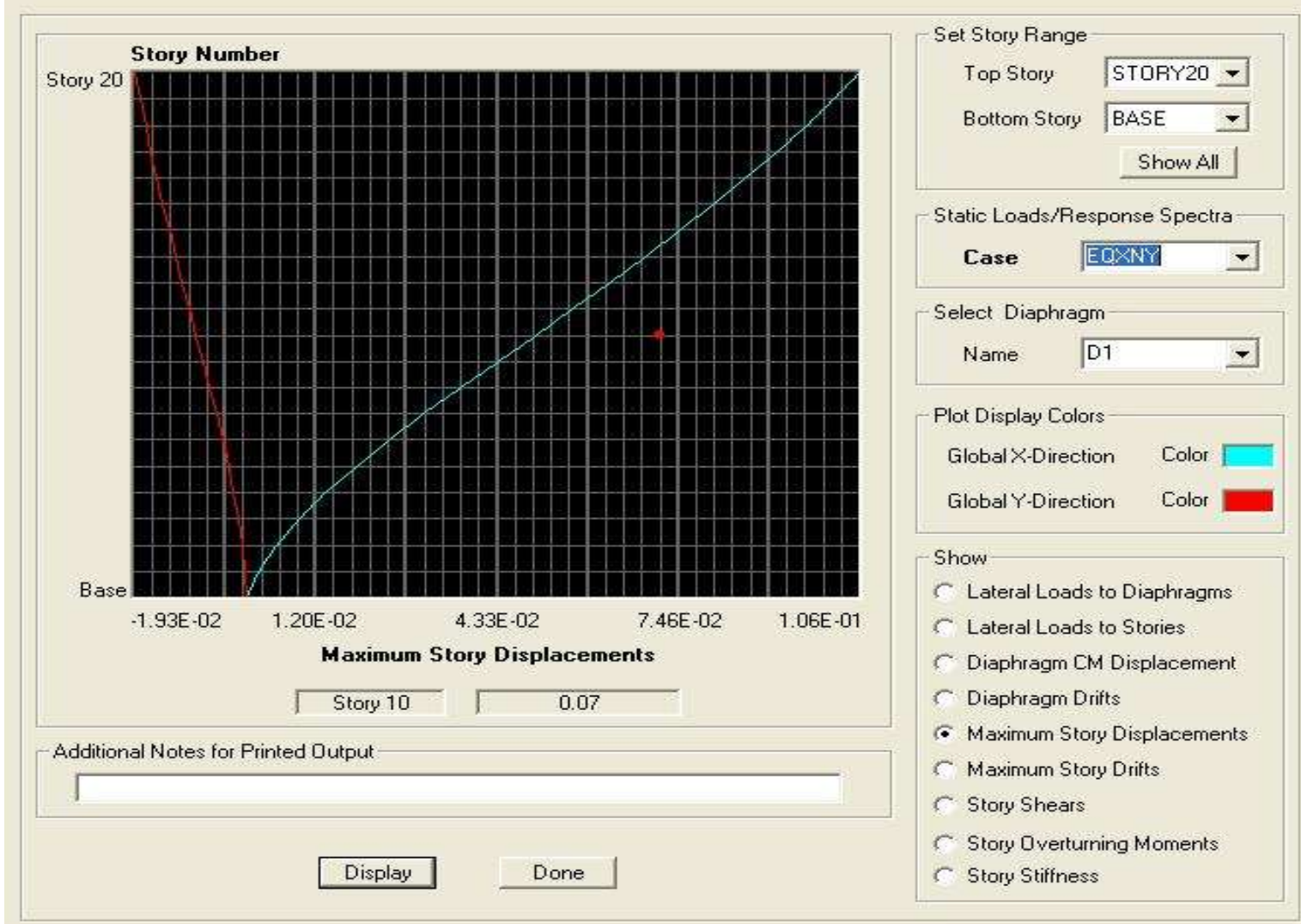

Graph 10: Displacement Opening 
DOI : https://dx.doi.org/10.26808/rs.ed.i8v5.03

International Journal of Emerging Trends in Engineering and Development

Issue 8, Vol.5 (Aug-Sep 2018)

Available online on http://www.rspublication.com/ijeted/ijeted_index.htm

ISSN 2249-6149

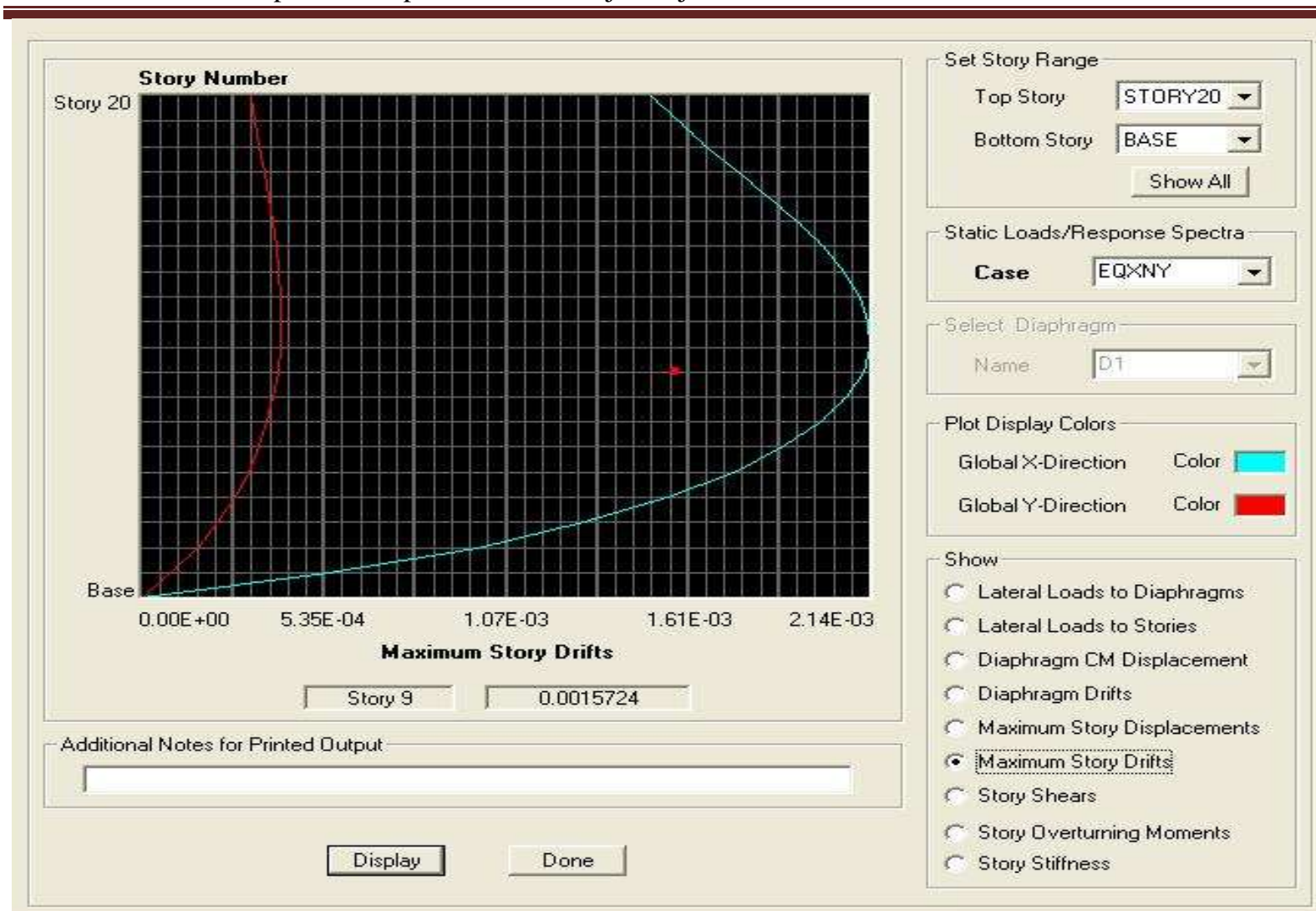

Graph 11: Drift Shear Wall

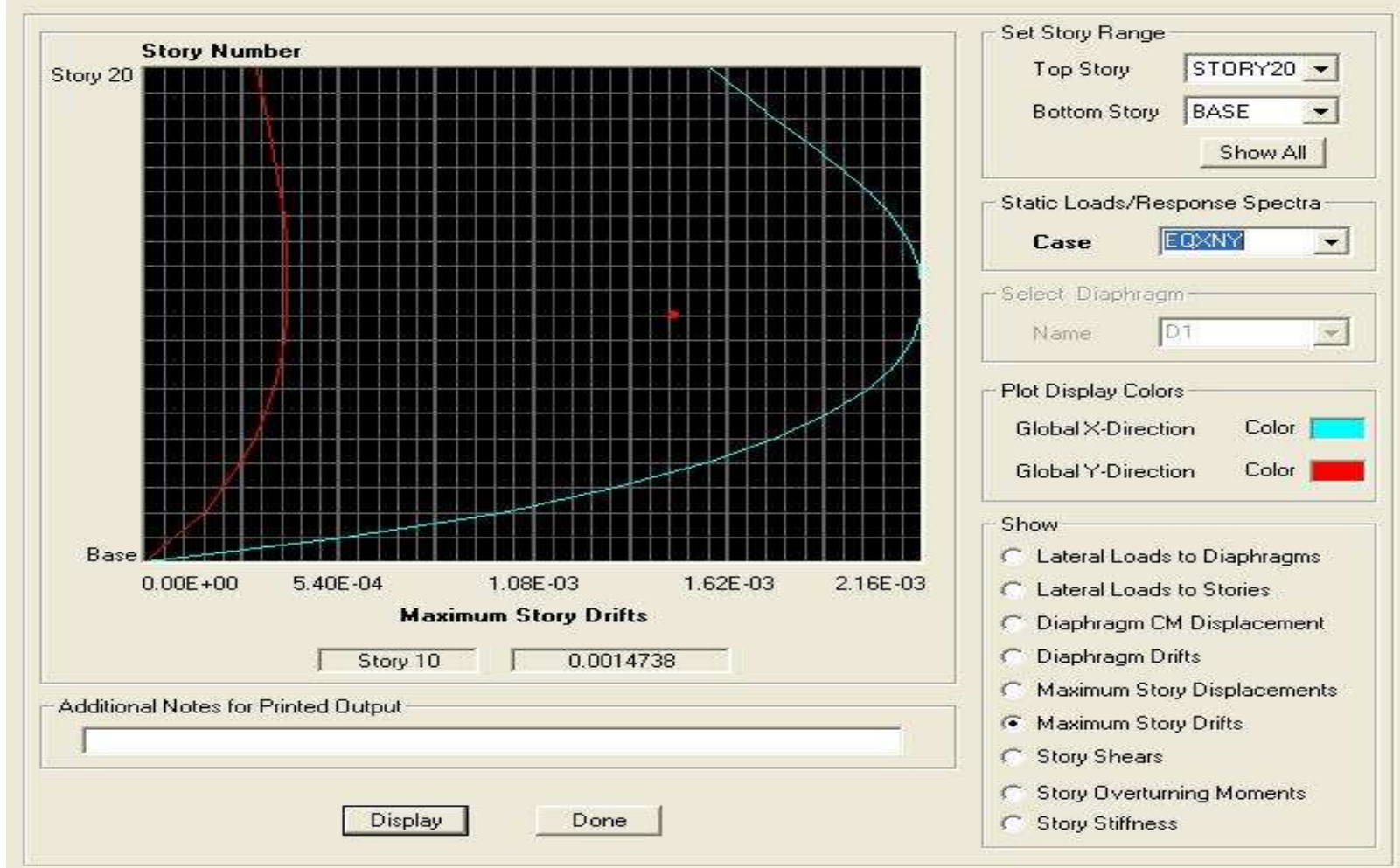

Graph 12: Drift With Opening 
DOI : https://dx.doi.org/10.26808/rs.ed.i8v5.03

International Journal of Emerging Trends in Engineering and Development

Issue 8, Vol.5 (Aug-Sep 2018)

Available online on http://www.rspublication.com/ijeted/ijeted_index.htm

ISSN 2249-6149

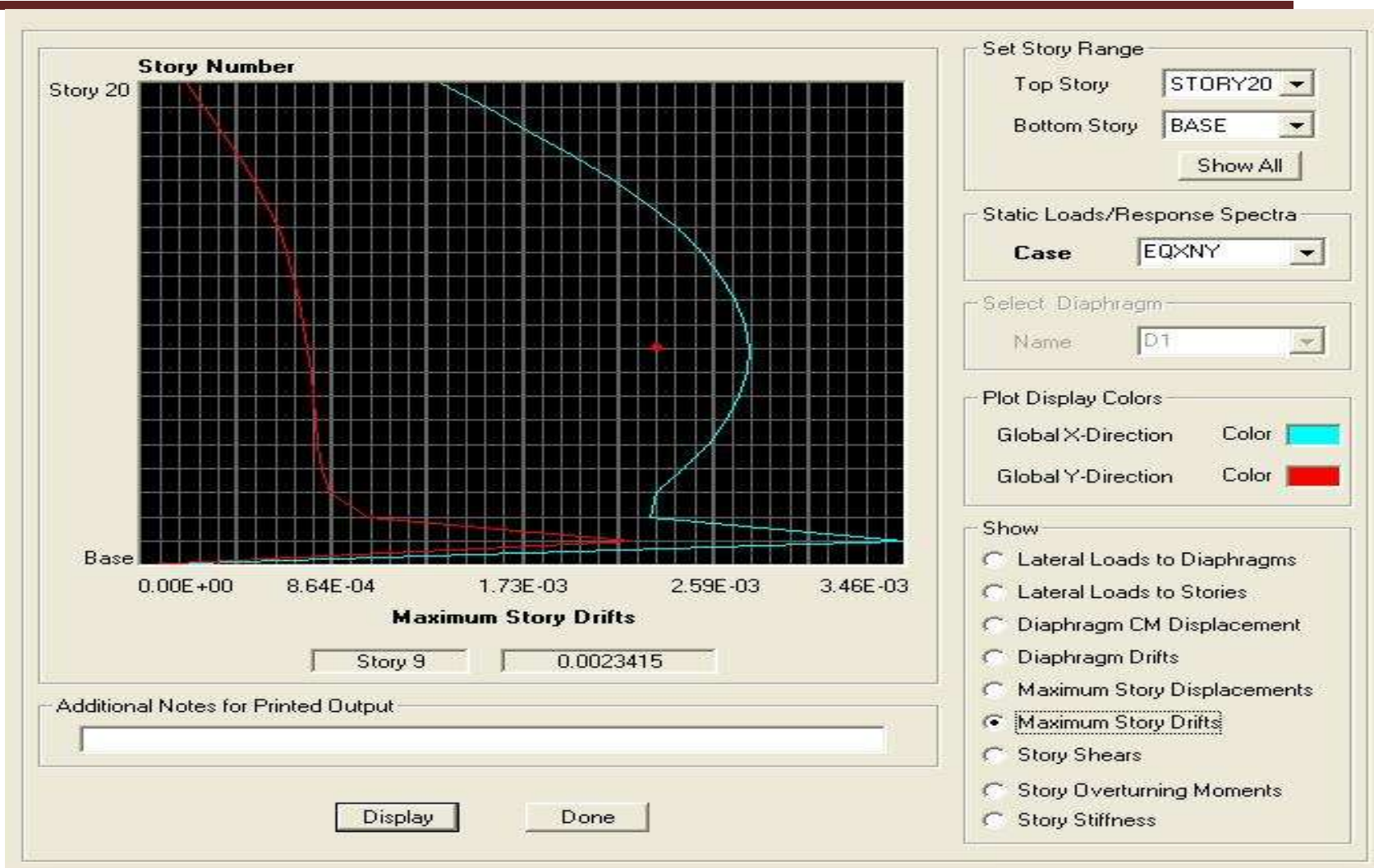

Graph 13: Drift With Out Shear Wall

\section{CONCLUSIONS}

$>$ With the provision of shear wall the shear force in the columns, decreased.

$>$ With the provision of shear wall the moment in the columns, increased.

$>$ No significant difference in shear force and moment provision of $20 \%$ opening in the shear wall.

$>$ With the provision of the shear walls drift and displacement is decreasing.

$>$ With the provision of the shear wall the drift and displacement is increasing.

\section{SCOPE FOR FURTHUR STUDY}

The size of opening and placement of the openings can be changed and a study can be made.

\section{REFERENCES}

[1] Bureau of Indian Standars, IS $456: 2000$, "Plain and Reinforced Concrete-Code of practice", New Delhi, India.

[2] Bureau of Indian Standards: IS 13920 : 1993, "Ductile detailing of reinforced concrete structures subjected to seismic forces - Code of Practice", New Delhi, India.

[3] Bureau of Indian Standards: IS 875( part 1) : 1987, "Dead loads on buildings and Structures", New Delhi, India. 
[4] Bureau of Indian Standards: IS 875( part 2 ) : 1987, "Live loads on buildings and Structures",New Delhi, India.

[5] Bureau of Indian Standards: IS 1893 (part 1) : 2002, "Criteria for earthquake resistant design of structures: Part 1 General provisions and buildings", New Delhi, India

[6] Ono, M. and Tokuhiro, I. (1992). A Proposal of Reducing Rate for Strength due to Opening Effect of Reinforced Concrete Framed Shear Walls. Journal of structural and construction engineering. No.435. 119-129.

[7] Ali, A. \& Wight, J. K. 1991. RC Structural Walls with Staggered Door Openings, Journal of Structural Engineering, ASCE, and Vol 117(5): 1514-1531.

[8] Collins M. P. \& Mitchell D. 1986. A Rational Approach to Shear Design - the 1984 Canadian Code Provisions, ACI Structural Journal, Vol 83(6): 925-933.

[9] Park, R. \& Paulay, T. 1975. Reinforced Concrete Structures, New York: John Wiley and Sons.

[10] Paulay, T. \& Priestley, M. J. N. 1992. Seismic Design of Reinforced Concrete and Masonry Buildings, New York: John Wiley.

[11] Schlaich, J., Schäfer, K. \& Jennewein, M. 1987. Toward a Consistent Design of Structural Concrete, PCI Journal, Vol 32(3): 74-150.

[12] Yanez, F. V., Park, R. \& Paulay, T. 1991. Seismic Behaviour of Reinforced Concrete Structural Walls with Irregular Openings, Pacific Conference on Earthquake Engineering, New Zealand.

[13] Yanez, F. V. 1993. Seismic Behaviour of Reinforced Concrete Walls with Irregular Openings, PhD dissertation, University of Canterbury, Christchurch, New Zealand. 